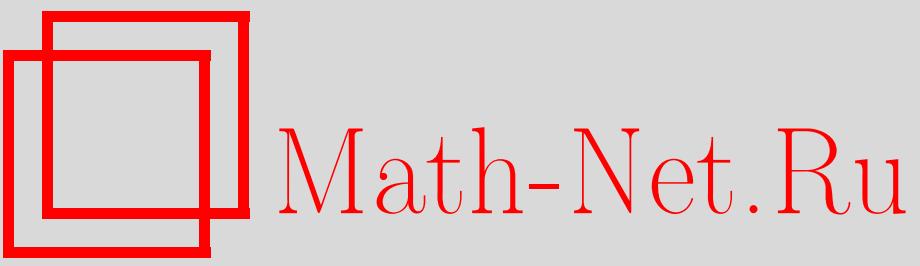

А. Д. Брюно, Асимптотики и разложения решений обыкновенного дифференциального уравнения, УМН, 2004, том 59, выпуск 3, 31-80

DOI: https://doi.org/10.4213/rm736

Использование Общероссийского математического портала Math-Net.Ru подразумевает, что вы прочитали и согласны с пользовательским соглашением

http://www.mathnet.ru/rus/agreement

Параметры загрузки:

IP : 54.224 .135 .184

26 апреля 2023 г., 12:44:40 


\title{
АСИМПТОТИКИ И РАЗЛОЖЕНИЯ РЕШЕНИЙ ОБЫКНОВЕННОГО ДИФФЕРЕНЦИАЛЬНОГО УРАВНЕНИЯ
}

\author{
А. Д. Брюно
}

Рассматривается обыкновенное дифференциальное уравнение весьма общего вида. Показывается, как с помощью алгоритмов степенной геометрии вблизи любого конечного или бесконечного значения независимой переменной находить: (а) все степенные асимптотики решений такого уравнения; (b) все степенно-логарифмические разложения решений, имеющих степенную асимптотику; (c) все нестепенные (экспоненциальные и логарифмические) асимптотики решений такого уравнения; (d) некоторые экспоненциально малые добавки к степенно-логариффмическому разложению решения, соответствующие экспоненциально близким решениям. Кроме теории и алгоритмов приводятся примеры вычислений указанных объектов для одного и того же уравнения. Основной упор делается на объяснение алгоритмов таких вычислений.

Библиография: 42 названия.

\section{СОДЕРЖАНИЕ}

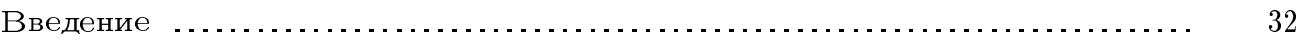

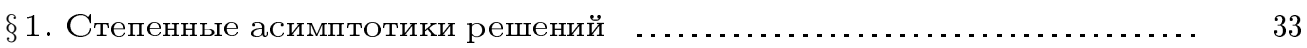

$\S 2$. Примеры вычисления степенных асимптотик .................... 39

$\S 3$. Степенно-логариффмические разложения решений $\ldots \ldots \ldots \ldots \ldots \ldots \ldots . .43$

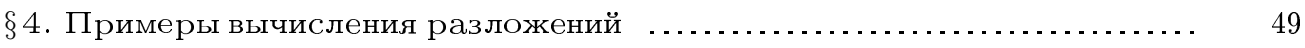

$\S 5$. Нестепенные асимптотики решений ............................ 56

$\S 6$. Примеры вычисления нестепенных асимптотик $\ldots \ldots \ldots \ldots \ldots \ldots \ldots \ldots .62$

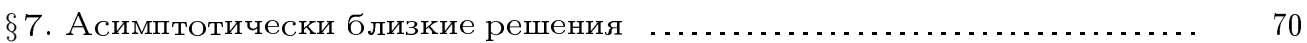

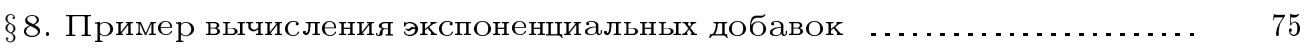

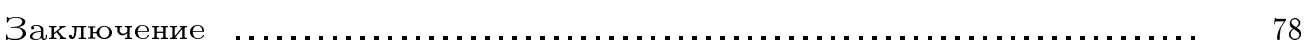

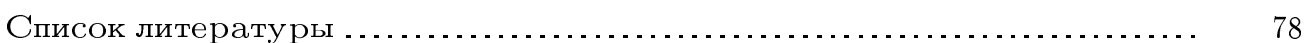

Работа выполнена при финансовой поддержке Российского фонда фундаментальных исследований (грант № 02-01-01067). 


\section{Введение}

В классическом анализе для решений регулярных (квазилинейных и аналитических) уравнений были получены локальные разложения по целым степеням независимой переменной (теоремы Коши о неявной функции и о решениях обыкновенных дифференциальных уравнений). В более сложных случаях получались локальные разложения решений по дробным степеням независимой переменной (ряды Пюизё). Теперь на основе степенной геометрии разработаны общие алгоритмы, позволяющие вычислять асимптотики решений, а также локальные асимптотические разложения решений существенно нелинейных или сингулярных уравнений. Оказалось, что для широкого круга дифференциальных уравнений эти разложения являются степенно-логарифмическими, т.е. по комплексным степеням независимой переменной с коэффициентами, являющимися многочленами от логарифмов этой переменной. И если для алгебраических кривых типичным является разложение по дробным степеням, то для решений обыкновенных дифференшиальных уравнений типичным является указанное степенно-логарифмическое разложение. Однако встречаются решения более сложной структуры, у которых указанное степенно-логарифмическое разложение имеется только для кратного логарифма решения.

В настоящей статье рассматривается обыкновенное дифференциальное уравнение

$$
f\left(x, y, y^{\prime}, \ldots, y^{(n)}\right)=0
$$

весьма общего вида, где $x$ - независимая, а $y$ - зависимая переменные. Показьвается, как с помощью алгоритмов степенной геометрии при $x \rightarrow 0$ или $\infty$ находить:

a) все степенные асимптотики решений $y(x)$ такого уравнения $(\S \S 1,2)$;

b) все степенно-логарифмические разложения решений, имеюших степенную асимптотику $(\S \S 3,4)$;

c) все нестепенные (экспоненциальные и логарифмические) асимптотики решений такого уравнения $(\S \S 5,6)$;

d) некоторые экспоненциально малые добавки к степенно-логарифмическому разложению решения, соответствуюшие экспоненциально близким решениям $(\S \S 7,8)$.

При этом в $\S \S 1,3,5,7$ излагаются соответствуюшая теория и алгоритмы, а в $\S \S 2,4,6,8$ даны примеры вычис лений указанных объектов для одного и того же уравнения. Основной упор делается на объяснение алгоритмов вычисления. Поэтому не для всех утверждений даны полные доказательства. Поскольку тут только две малые или большие координаты, то используется только двумерная степенная геометрия, иллюстрированная 15 рисунками, что, как надеется автор, делает изложение геометрически наглядным и более понятным. Похожее изложение (для других задач) см. в главах I и II книги [1].

Заметим, что далеко не все задачи, имеюшиеся для обыкновенного дифференциального уравнения, решаются методами, излагаемыми в настоящей статье. Таковой является, например, проблема различения центра и фокуса $[1 ;$ гл. II, $\S 4]$. В ней излагаемые здесь методы позволяют найти только комплексные сепаратрисы, которых не достаточно для решения этой проблемы. 


\section{$\S 1$. Степенные асимптотики решений}

1.1. Постановка задачи. Сначала напомним некоторые понятия степенной геометрии [1], [2]. Пусть $x$ - независимая и $y$ - зависимая переменные, $x, y \in \mathbb{C}$. Положим $X=(x, y)$. Дифференциальным мономом $a(x, y)$ называется произведение обыгного монома

$$
c x^{r_{1}} y^{r_{2}} \stackrel{\text { def }}{=} c X^{R}
$$

где $c=$ const $\in \mathbb{C}, R=\left(r_{1}, r_{2}\right) \in \mathbb{R}^{2}$, и конечного числа производных вида

$$
d^{l} y / d x^{l}, \quad l \in \mathbb{N}
$$

Сумма дифференциальных мономов

$$
f(X)=\sum a_{i}(X)
$$

называется дифференииальной суммой.

Пусть задано обыкновенное дифференциальное уравнение

$$
f(X)=0
$$

где $f(X)$ - дифференциальная сумма, в которую $y$ входит в цельх степенях. Положим

$$
\omega= \begin{cases}-1, & \text { если } x \rightarrow 0, \\ 1, & \text { если } x \rightarrow \infty .\end{cases}
$$

Пусть $x \rightarrow 0$ или $\infty$ и решение уравнения (1.4) имеет вид

$$
y=c_{r} x^{r}+o\left(|x|^{r+\varepsilon}\right),
$$

где коэффициент $c_{r}=$ const $\in \mathbb{C}, c_{r} \neq 0$, показатели степени $r, \varepsilon \in \mathbb{R}$ и $\varepsilon \omega<0$. Тогда выражение

$$
y=c_{r} x^{r}, \quad c_{r} \neq 0
$$

является степенной асимптотикой решения (1.6).

ЗАДАчА 1. Для заданного уравнения (1.4) найти все степенные асимптотики (1.7) его решений вида (1.6).

Для решения задачи 1 степенная геометрия дает теорию и алгоритмы, основанные на выделении укороченных уравнений.

1.2. Выделение укороченных уравнений. Каждому дифференциальному моному $a(X)$ ставится в соответствие его (векторньй) показатель степени $Q(a)=$ $\left(q_{1}, q_{2}\right) \in \mathbb{R}^{2}$ по следуюшим правилам. Для монома вида (1.1)

$$
Q\left(c X^{R}\right)=R
$$


т.е. $Q\left(c x^{r_{1}} y^{r_{2}}\right)=\left(r_{1}, r_{2}\right)$; для производной $(1.2)$

$$
Q\left(d^{l} y / d x^{l}\right)=(-l, 1)
$$

при умножении дифференциальных мономов их показатели степени складьваются как векторы:

$$
Q\left(a_{1} a_{2}\right)=Q\left(a_{1}\right)+Q\left(a_{2}\right) .
$$

Множество $\mathbf{S}(f)$ показателей степеней $Q\left(a_{i}\right)$ всех дифференциальных мономов $a_{i}(X)$, входящих в дифференциальную сумму (1.3), назьвается носителем суммы $f(X)$. Очевидно, $\mathbf{S}(f) \in \mathbb{R}^{2}$. Через $f_{Q}(X)$ обозначим сумму тех мономов $a_{i}(X)$ из (1.3), у которых $Q\left(a_{i}\right)=Q$. Тогда дифференциальную сумму (1.3) можно записать в виде

$$
f(X)=\sum f_{Q}(X) \text { по } Q \in \mathbf{S}(f)
$$

Замыкание вьпуклой оболочки $\Gamma(f)$ носителя $\mathbf{S}(f)$ назьвается многоугольником cyммы $f(X)$. Граница $\partial \Gamma(f)$ многоугольника $\Gamma(f)$ состоит из вершин $\Gamma_{j}^{(0)}$ и ребер $\Gamma_{j}^{(1)}$. Их назьвают (обобшенными) гранями $\Gamma_{j}^{(d)}$, где верхний индекс указьвает размерность грани, а нижний - ее номер. Каждой грани $\Gamma_{j}^{(d)}$ соответствуют граничное подмножество $\mathbf{S}_{j}^{(d)}=\mathbf{S}(f) \cap \Gamma_{j}^{(d)}$ множества $\mathbf{S}$ и укороченная сумма

$$
\hat{f}_{j}^{(d)}(X)=\sum a_{i}(X) \text { по } Q\left(a_{i}\right) \in \mathbf{S}_{j}^{(d)}
$$

Пусть плоскость $\mathbb{R}_{*}^{2}$ сопряжена плоскости $\mathbb{R}^{2}$ так, что для $P=\left(p_{1}, p_{2}\right) \in \mathbb{R}_{*}^{2}$ и $Q=$ $\left(q_{1}, q_{2}\right) \in \mathbb{R}^{2}$ определено скалярное произведение

$$
\langle P, Q\rangle \stackrel{\text { def }}{=} p_{1} q_{1}+p_{2} q_{2} .
$$

Каждой грани $\Gamma_{j}^{(d)}$ в плоскости $\mathbb{R}_{*}^{2}$ соответствуют: свое нормальное подпространство

$$
\mathbf{N}_{j}^{(d)}=\left\{P:\langle P, Q\rangle=\left\langle P, Q^{\prime}\right\rangle \text { по } Q, Q^{\prime} \in \mathbf{S}_{j}^{(d)}\right\}
$$

и свой нормальный конус

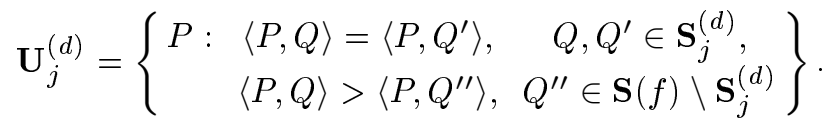

Пусть вектор $N_{j}$ - это внешняя нормаль к ребру $\Gamma_{j}^{(d)}$. Для ребра $\Gamma_{j}^{(1)}$ нормальное подпространство $\mathbf{N}_{j}^{(1)}$ - это прямая, проходящая через начало координат $P=0$ и точку $P=N_{j}$, а нормальньй конус - это тот луч прямой $\mathbf{N}_{j}^{(1)}$, который направлен от ребра $\Gamma_{j}^{(1)}$ наружу многоугольника $\Gamma(f)$, т.е. натянут на вектор $N_{j}$. Для вершины $\Gamma_{j}^{(0)}$ нормальное подпространство $\mathbf{N}_{j}^{(0)}=\mathbb{R}_{*}^{2}$, а нормальный конус $\mathbf{U}_{j}^{(0)}$ - это открытьй сектор (угол) на плоскости $\mathbb{R}_{*}^{2}$ с вершиной в нуле $P=0$, ограниченньй лучами, являющимися нормальными конусами ребер, примыкаюших к вершине $\Gamma_{j}^{(0)}$. 
ПРЕДЛОЖЕНИЕ 1.1. Если носитель $\mathbf{S}(f)$ состоит из одной вериины $\Gamma_{1}^{(0)}$, то нормальный конус $\mathrm{U}_{1}^{(0)}=\mathbb{R}_{*}^{2}$, m.е. в $\mathrm{U}_{1}^{(0)}$ лежат оба вектора $\pm R$. В противном случае раствор угла $\mathrm{U}_{j}^{(0)}$ меньше $\pi$, т.е. из двух векторов $\pm R$ в нормальныи конус $\mathbf{U}_{j}^{(0)}$ попадает только один. Здесь вектор $R \neq 0, R \in \mathbb{R}_{*}^{2}$.

ПРЕДЛОЖЕНИЕ 1.2. Если многоугольник $Г(f)$ состоит из одного ребра $\Gamma_{1}^{(1)}$, то нормальный конус $\mathrm{U}_{1}^{(1)}$ - это прямая, т.е. в $\mathrm{U}_{1}^{(1)}$ лежсат оба вектора $\pm R$. $B$ противном случае в нормальном конусе $\mathrm{U}_{j}^{(1)}$ лежит только один из векторов $\pm R$. Здесь вектор $R \neq 0$ является нормалью к ребру $\Gamma_{j}^{(1)}$.

Итак, каждой грани $\Gamma_{j}^{(d)}$ соответствуют: нормальное подпространство $\mathbf{N}_{j}^{(d)}$ и нормальный конус $\mathbf{U}_{j}^{(d)}$ в $\mathbb{R}_{*}^{2}$ и укороченное уравнение

$$
\hat{f}_{j}^{(d)}(X)=0
$$

Носитель степенной асимптотики (1.7) состоит из двух точек $E_{2} \stackrel{\text { def }}{=}(0,1)$ и $(r, 0)$. Их вьпуклая оболочка является ребром, которое обозначим $\gamma_{1}^{(1)}$. Его нормальное подпространство $\mathbf{n}$ является прямой, натянутой на вектор $(1, r)$. Нормальнылм конусом и решения вида (1.6) назовем луч $\lambda \omega(1, r)$, где $\omega$ определено по $(1.5)$ и $\lambda>0$.

Теорема 1.1 [2; гл. VI, теорема 1.1]. Если уравнение (1.4) имеет решение (1.6) $u \mathbf{u} \in \mathbf{U}_{j}^{(d)}$, то укорочение (1.7) решения (1.6) является решением укороченного уравнения (1.8), (1.9).

Поэтому для нахождения всех укороченных решений (1.7) уравнения (1.4) надо вычислить: носитель $\mathbf{S}(f)$, многоугольник $\Gamma(f)$, все его грани $\Gamma_{j}^{(d)}$, внешние нормали $N_{j}$ к ребрам $\Gamma_{j}^{(1)}$, нормальные конусы ребер $\mathbf{U}_{j}^{(1)}$ и нормальные конусы вершин $\mathbf{U}_{j}^{(0)}$. Затем для каждого укороченного уравнения (1.8), (1.9) надо найти все его решения (1.7), у которых вектор $(1, r)$ лежит в $\mathbf{N}_{j}^{(d)}$, и из них отобрать те, у которых один из векторов $\pm(1, r)$ лежит в нормальном конусе $\mathbf{U}_{j}^{(d)}$. Если $d=0$, то это означает, что один из векторов $\pm(1, r)$ лежит в $\mathbf{U}_{j}^{(d)}$. Если $d=1$, то это свойство всегда вьполнено. При этом согласно предложениям 1.1 и 1.2 определяется также значение $\omega$.

1.3. Решение укороченного уравнения. Здесь рассмотрим по отдельности два случая: вершины $\Gamma_{j}^{(0)}$ и ребра $\Gamma_{j}^{(1)}$.

Вершине $\Gamma_{j}^{(0)}=\{Q\}$ соответствует укороченное уравнение (1.9) с точечным носителем $Q$ и с $d=0$. Положим $g(X) \stackrel{\text { def }}{=} X^{-Q} \hat{f}_{j}^{(0)}(X)$, тогда решение $(1.7)$ уравнения (1.9) удовлетворяет уравнению

$$
g(X)=0
$$

Подставляя $y=c x^{r}$ в $g(X)$, получаем, что $g\left(x, c x^{r}\right)$ не зависит от $x$ и $c$ и является многочленом от $r$, т.е.

$$
g\left(x, c x^{r}\right) \equiv \chi(r)
$$


где $\chi(r)$ - характеристический многочлен дифференциальной суммы $\hat{f}_{j}^{(0)}(X)$. Следовательно, для решения (1.7) уравнения (1.9) показатель $r$ является корнем характеристического уравнения

$$
\chi(r) \stackrel{\text { def }}{=} g\left(x, x^{r}\right)=0,
$$

а коэффициент $c_{r}$ - произвольный. Из корней $r_{i}$ уравнения (1.10) надо отобрать только те, для которых один из векторов $\omega(1, r)$, где $\omega= \pm 1$, лежит в нормальном конусе $\mathrm{U}_{j}^{(0)}$ вершины $\Gamma_{j}^{(0)}$. При этом согласно предложению 1.1 значение $\omega$ определяется однозначно. Соответствующие выражения (1.7) с произвольной константой $c_{r}$ являются кандидатами на роль укороченных решений уравнения (1.4). При этом согласно (1.5) если $\omega=-1$, то $x \rightarrow 0$, а если $\omega=1$, то $x \rightarrow \infty$.

Укороченное уравнение (1.9) назьвается алгебраическим, если оно не содержит производных.

ЗАмЕчАниЕ 1.1 [2]. Если укороченное уравнение (1.9) с $d=0$ является алгебраическим, то оно не имеет решений вида (1.7). Поэтому укорочения, состояшие из одного алгебраического монома, можно не рассматривать.

Ребру $\Gamma_{j}^{(1)}$ соответствует укороченное уравнение $(1.9)$ с $d=1$, нормальньй конус $\mathbf{U}_{j}^{(1)}$ которого является лучом $\left\{\lambda N_{j}, \lambda>0\right\}$. Нормальньй конус $\mathbf{u}$ укороченного решения (1.7) пересекается с $\mathbf{U}_{j}^{(1)}$ только если вектор $\omega(1, r) \in \mathbf{U}_{j}^{(1)}$. Согласно предложению 1.2 этим однозначно определяются показатель степени $r$ укороченного решения (1.7) и значение $\omega= \pm 1$ в (1.5). Для определения коэффициента $c_{r}$ надо выражение (1.7) подставить в укороченное уравнение (1.9). После сокрашения на некоторую степень $x$ получаем алгебраическое определяющее уравнение для коэффициента $c_{r}$

$$
\tilde{\tilde{f}}\left(c_{r}\right) \stackrel{\text { def }}{=} x^{-s} \hat{f}_{j}^{(1)}\left(x, c_{r} x^{r}\right)=0 .
$$

Каждому его корню $c_{r}=c_{r}^{(i)} \neq 0$ соответствует свое выражение $(1.7)$, которое является кандидатом на роль укороченного решения уравнения (1.4). При этом согласно $(1.5)$ если в нормальном конусе $\mathbf{U}_{j}^{(1)}$ координата $p_{1}<0$, то $x \rightarrow 0$, а если $p_{1}>0$, то $x \rightarrow \infty$.

Итак, каждое укороченное уравнение (1.9) имеет несколько подходящих решений (1.7) с $\mathbf{u} \subset \mathbf{U}_{j}^{(d)}$. Объединим их в непрерывные по $\omega, r, c_{r}$ и параметрам уравнения (1.4) семейства, которые обозначим $\mathscr{F}_{j}^{(d)} k$, где $k=1,2, \ldots$

Если нас интересуют не все решения (1.6) уравнения (1.4), а только те, у которых нормальный конус и лежит в некотором заданном конусе $\mathscr{K}$, то $\mathscr{K}$ назьвается конусом задачи [2; гл. I, §6]. Например, для укороченного уравнения (1.9) нормальный конус $\mathbf{U}_{j}^{(d)}$ является конусом задачи, если нет других ограничений.

1.4. Критические числа укороченного решения. Если найдено укороченное решение (1.7), то замена

$$
y=c_{r} x^{r}+z
$$

приводит уравнение (1.4) к виду

$$
\tilde{f}(x, z) \stackrel{\text { def }}{=} f\left(x, c x^{r}+z\right)=0,
$$


где $\tilde{f}(x, z)$ - дифференциальная сумма, все точки $Q=\left(q_{1}, q_{2}\right)$ ее носителя $\mathbf{S}(\tilde{f})$ имеют целую неотрицательную координату $q_{2}$. K уравнению (1.13) можно применить описанные вьше вычисления (т.е. вычисления носителя, многоугольника, укорочений и т. д.) и получить для решения (1.6) следуюший член разложения $c_{k_{0}} x^{k_{0}}$, у которого $k_{0}>r$, если $x \rightarrow 0$, и $k_{0}<r$, если $x \rightarrow \infty$. Следовательно, получилась задача 1 для уравнения (1.13), но теперь с конусом задачи

$$
\mathscr{K}=\left\{P=\left(p_{1}, p_{2}\right): \begin{array}{l}
p_{2} / p_{1}>r, p_{1}<0, \text { если } x \rightarrow 0 \\
p_{2} / p_{1}<r, p_{1}>0,
\end{array}\right.
$$

Согласно (1.5) для $k=p_{2} / p_{1}$ этот конус задачи можно записать в виде

$$
\mathscr{K}=\left\{k=p_{2} / p_{1}: k \omega<r \omega, p_{1} \omega>0\right\} .
$$

Однако во многих случаях дифференциальная сумма $\tilde{f}(x, z)$ имеет специальньй вид, что позволяет сушественно сократить вычисления разложений решения (1.6). Предположим, что уравнение (1.13) имеет вид

$$
\tilde{f}(x, z) \stackrel{\text { def }}{=} \mathscr{L}(x) z+h(x, z)=0
$$

где $\mathscr{L}(x)$ - линейный дифференциальньй оператор, и носитель $\mathbf{S}(\mathscr{L} z)$ состоит из одной точки $(v, 1)$, являющейся вершиной $\widetilde{\Gamma}_{1}^{(0)}$ многоугольника $\Gamma(\tilde{f})$, у всех точек $Q=$ $\left(q_{1}, q_{2}\right)$ носителя $\mathbf{S}(h)$ координата $q_{2} \geqslant 0$ и нет точки $Q=(v, 1)$, нормальньй конус вершины $\widetilde{\Gamma}_{1}^{(0)}$ содержит вектор $P=\left(p_{1}, p_{2}\right)$ с $p_{1} \omega>0$.

По аналогии с известной в функциональном анализе производной Фреше [42] мы введем формальную производную Фреше (или первую вариацию) $\delta f(x, y) / \delta y$ дифференциальной суммы $f(x, y)$, которая обладает следуюшими свойствами и определяется ими:

$$
\begin{gathered}
\delta\left(c x^{q_{1}} y^{q_{2}}\right) / \delta y=c q_{2} x^{q_{1}} y^{q_{2}-1}, \\
\delta\left(d^{l} y / d x^{l}\right) / \delta y=d^{l} / d x^{l}, \\
\delta(f+g) / \delta y=\delta f / \delta y+\delta g / \delta y, \\
\delta(f g) / \delta y=(\delta f / \delta y) g+f(\delta g / \delta y) .
\end{gathered}
$$

В дальнейшем изложении прилагательное “формальная" ради краткости опускается. Согласно второму свойству производная Фреше - это линейньй дифференциальный оператор, т.е. имеет вид

$$
\sum_{k=0}^{l} g_{k}(x, y) \frac{d^{k}}{d x^{k}},
$$

где $g_{k}(x, y)$ суть дифференциальные суммы. 
ТеОРема 1.2 [3]-[5]. Пусть (1.7) - решение укороченного уравнения (1.9) $с \mathbf{u} \in$ $\mathrm{U}_{j}^{(d)}$. Тогда в уравнении (1.15) оператор

$$
\mathscr{L}(x)=\frac{\delta \hat{f}_{j}^{(d)}(x, y)}{\delta y} \quad \text { на } \quad y=c_{r} x^{r},
$$

т.е. равен первой вариации, вычисленной на решении (1.7). При этом $\mathbf{S}(\mathscr{L} z)=$ $(v, 1)$, əде $v=\left\langle Q_{1},(1, r)\right\rangle-r c Q_{1} \in \Gamma_{j}^{(d)}$.

Следовательно, после подстановки (1.13) уравнение (1.4) принимает вид (1.15), если

$$
\mathscr{L}(x) \not \equiv 0 .
$$

Пусть $\nu(k)$ - характеристический многочлен дифференциальной суммы $\mathscr{L}(x) z$, т.е.

$$
\nu(k)=x^{-v-k} \mathscr{L}(x) x^{k} .
$$

Если $\nu(k) \not \equiv 0$, то корни $k_{1}, \ldots, k_{s}$ многочлена $\nu(k)$ называются собственнылми значениями укороченного решения (1.7). Те из вещественных собственных чисел $k_{i}$, которые лежат в конусе задачи, т.е. удовлетворяют неравенствам (1.14), назьваются критическими числами. Они играют важную роль при нахождении разложения решения (1.6), что будет показано в $\S 3$.

ЗАмечАниЕ 1.2. Степенное решение (1.7) алгебраического укороченного уравнения (1.9) с $d=1$ не имеет собственных значений и критических чисел, ибо для него

$$
\nu(k) \equiv \nu_{0}=\mathrm{const}=\frac{\partial \hat{f}_{j}^{(1)}}{\partial y}\left(1, c_{r}\right) .
$$

Если $c_{r}-$ простой корень уравнения $(1.11)$, то $\nu_{0} \neq 0$. Если $c_{r}-$ кратньй корень уравнения (1.11), то $\nu_{0}=0$.

Если $\mathscr{L}(x) \not \equiv 0$, то $\nu(k) \not \equiv 0$. Если же $\mathscr{L}(x) \equiv 0$, то для уравнения (1.13) надо решать задачу 1 , как описано в пш. 1.2 и 1.3 с учетом конуса задачи (1.14).

1.5. Решения с комплексными показателями степени. В задаче 1 п. 1.1 искались только решения вида (1.6) с вешественными показателями $r$. Теперь рассмотрим решения вида

$$
y=c_{\rho} x^{\rho}+o\left(|x|^{r+\varepsilon}\right), \quad c_{\rho} \neq 0,
$$

с комплексным показателем $\rho=r+i s$, где $\varepsilon \omega<0$. Их асимптотики имеют вид

$$
y=c_{\rho} x^{\rho}, \quad c_{\rho} \neq 0 .
$$

Для таких решений справедливо все сказанное в предыдуших пунктах, если $r=\operatorname{Re} \rho$.

Уточним только, что решения вида (1.19) укороченного уравнения (1.9) возникают только для вершин $\Gamma_{j}^{(0)}$, т.е. для $d=0$. В этом случае характеристическое уравнение (1.10) может иметь комплексные корни, которые обозначим $\rho_{1}, \ldots, \rho_{l}$. Подходящим корнем $\rho_{i}$ является такой, для которого один из векторов $\pm\left(1, \operatorname{Re} \rho_{i}\right)$ лежит в нормальном конусе $\mathbf{U}_{j}^{(0)}$. Если вместо (1.12) сделать замену

$$
y=c_{\rho} x^{\rho}+z,
$$


то дифференщиальная сумма $\tilde{f}(x, z) \stackrel{\text { def }}{=} f\left(x, c_{\rho} x^{\rho}+z\right)$ будет содержать обычные мономы вида $c x^{r_{1}} z^{r_{2}}$ с комплексными $r_{1}$. Теорема 1.2 сохраняется, только производную $\Phi$ реше надо вычислять на решении (1.19). Характеристический многочлен $\nu(k)$ также определяется по (1.17). Его корни $k_{i}$ также являются собственными значениями укороченного решения (1.19). Те из них, у которых вешественные части $\operatorname{Re} k_{i}$ лежат в конусе задачи, т.е. удовлетворяют неравенствам (1.14), назьваются критическими числами укороченного решения (1.19).

Для укороченного уравнения (1.9), соответствующего ребру $\Gamma_{j}^{(1)}$, ищутся только решения (1.7) с вешественным показателем $r$, который однозначно определяется нормалью к этому ребру.

\section{§ 2. Примеры вычисления степенных асимптотик}

Методами $§ 1$ были найдены степенные асимптотики решений первого уравнения Пенлеве $-y^{\prime \prime}+6 y^{2}+x=0$ в [2; гл. VI, $\S 1$, пример 1.1$]$ и четвертого уравнения Пенлеве $-2 y y^{\prime \prime}+y^{2}+3 y^{4}+8 x y^{3}+4\left(x^{2}-a\right) y^{2}+2 b=0$ в [3; $\left.\S 7\right]$. Однако первые четыре уравнения Пенлеве [40] не имеют слишком сложных решений, они хороши для иллюстрации степенных разложений решений, но не пригодны для иллюстрации степенно-логарифмических разложений (см. [36], [24]). Пятое и шестое уравнения Пенлеве имеют достаточно сложные решения, но сами уравнения слишком громоздки, чтобы служить иллюстрацией (см. [25], [22]). Поэтому здесь в качестве примера рассмотрим уравнение

$$
f(X) \stackrel{\text { def }}{=} x^{2} y^{\prime 2}-2 x^{2} y y^{\prime \prime}+a y^{2}+x^{2} y^{2}-x^{4}=0,
$$

в котором $a$ - вешественньй параметр. В [2; гл. VI] вычислены степенные асимптотики решений уравнения Эмдена-Фаулера $y^{\prime \prime}=a x^{\sigma} y^{\mu}$ (примеры 1.2 и 2.1 ) и его обобшения $y^{\prime \prime \prime}=x^{\sigma} y^{\mu}(\S 4)$.

2.1. Многоугольник и нормальные конусы граней. Легко видеть, что в уравнении (2.1) $f(X)$ является дифференциальной суммой. Найдем показатели степеней $Q=\left(q_{1}, q_{2}\right)$ для ее дифференциальных мономов. Первые три монома имеют показатель $Q_{1}=(0,2)$, показатель четвертого монома есть $Q_{2}=(2,2)$, а у пятого он равен $Q_{3}=(4,0)$. Следовательно, носитель $\mathbf{S}(f)$ состоит из трех точек

$$
Q_{1}=(0,2), \quad Q_{2}=(2,2), \quad Q_{3}=(4,0),
$$

показанных на рис. 1. Их вьпуклая оболочка - это треугольник $\Gamma(f)$ с вершинами $Q_{1}, Q_{2}, Q_{3}$ (заштрихован на рис. 1). Его граница состоит из трех вершин $\Gamma_{j}^{(0)}=Q_{j}$, $j=1,2,3$, и трех ребер $\Gamma_{j}^{(1)}$ (см. рис. 1$)$. Ребро $\Gamma_{1}^{(1)}$ соединяет точки $Q_{1}$ и $Q_{3}$. Поскольку разность $Q_{1}-Q_{3}=(-4,2)=2(-2,1)$, то вектор $R=(-2,1)$ является направляюшим для этого ребра. Следовательно, нормальным к ребру $\Gamma_{1}^{(1)}$ является вектор $\widetilde{N}_{1}=(1,2)$ и нормальное подпространство $\mathbf{N}_{1}^{(1)}=\left\{P=\mu \widetilde{N}_{1}, \mu \in \mathbb{R}\right\}$. Но вектор $\widetilde{N}_{1}$ направлен от ребра $\Gamma_{1}^{(1)}$ внутрь многоугольника $\Gamma(f)$. Внешней же нормалью к ребру $\Gamma_{1}^{(1)}$ является вектор $N_{1}=-\widetilde{N}_{1}=(-1,-2)$. Следовательно, для ребра $\Gamma_{1}^{(1)}$ нормальный конус $\mathbf{U}_{1}^{(1)}=\left\{P=\lambda N_{1}, \lambda>0\right\}$, т.е. это луч, показанньй на рис. 2. Аналогично находим нормальные конусы

$$
\mathbf{U}_{j}^{(1)}=\left\{P=\lambda N_{j}, \lambda>0\right\}, \quad j=2,3,
$$


где

$$
N_{2}=(0,1), \quad N_{3}=(1,1) .
$$

Нормальньй конус $\mathbf{U}_{j}^{(0)}$ вершины $\Gamma_{j}^{(0)}$ - это сектор, ограниченньй лучами, которые являются нормальньми конусами ребер, примыкающих к этой вершине (рис. 2).

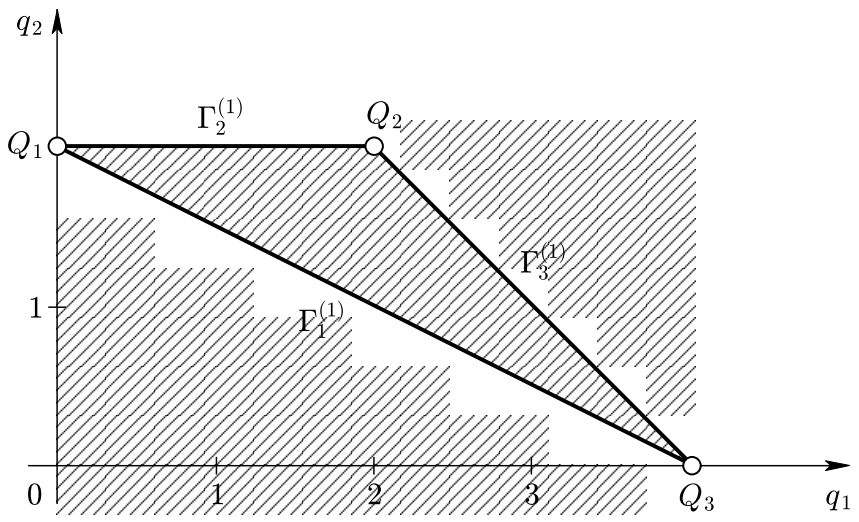

Рис. 1.

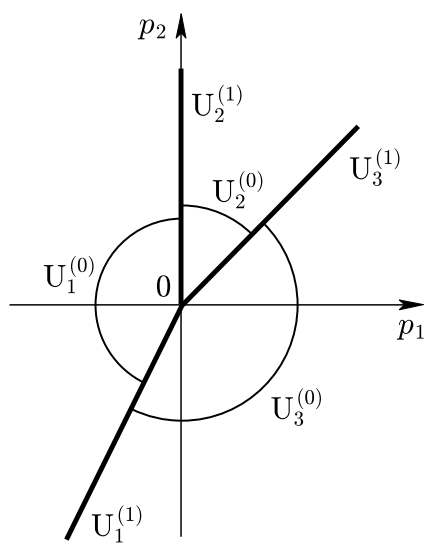

Рис. 2.

Теперь для каждой обобшенной грани $\Gamma_{j}^{(d)}$ найдем подходяшие укороченные решения (1.7) соответствуюшего укороченного уравнения (1.9) и критические числа решения (1.7).

2.2. Вершина $\Gamma_{1}^{(0)}$. Ей соответствует укороченное уравнение

$$
\hat{f}_{1}^{(0)}(X) \stackrel{\text { def }}{=} x^{2} y^{\prime 2}-2 x^{2} y y^{\prime \prime}+a y^{2}=0 .
$$

Согласно рис. 2 у всех векторов $P=\left(p_{1}, p_{2}\right) \in \mathbf{U}_{1}^{(0)}$ всегда $p_{1}<0$ и $p_{2} / p_{1}<2$. Поэтому здесь $x \rightarrow 0$ и $\omega=-1$. Подставляя $y=x^{\rho}$ в $\hat{f}_{1}^{(0)}(X)$ и сокрашая результат 
на $x^{2 \rho}$, получаем характеристическое уравнение

$$
\chi(\rho) \stackrel{\text { def }}{=} \rho^{2}-2 \rho(\rho-1)+a=-\left[\rho^{2}-2 \rho-a\right]=0 .
$$

Его корни

$$
\rho_{1,2}=1 \pm \sqrt{1+a}
$$

Если $a>-1$, то оба корня вещественны и различны, т.е. $r_{i}=\rho_{i}$. Если $a \in(-1,0)$, то оба вектора $-\left(1, \rho_{1}\right)$ и $-\left(1, \rho_{2}\right)$ лежат в $\mathbf{U}_{1}^{(0)}$. Если $a \in[0,+\infty)$, то в $\mathbf{U}_{1}^{(0)}$ лежит только один вектор $-\left(1, \rho_{2}\right)=(-1,-1+\sqrt{1+a})$. Итак, для $a>-1$ имеются два семейства подходяших степенных решений (1.7)

$$
\begin{aligned}
& \mathscr{F}_{1}^{(0)} 1=\left\{y=c x^{r_{1}}, \omega=-1, a \in(-1,0)\right\}, \\
& \mathscr{F}_{1}^{(0)} 2=\left\{y=c x^{r_{2}}, \omega=-1, a \in(-1,+\infty)\right\},
\end{aligned}
$$

где $c \neq 0$ - любая постоянная из $\mathbb{C}$.

Если $a=-1$, то характеристическое уравнение имеет двукратньй корень $\rho=1$ и вектор $-(1,1) \in \mathbf{U}_{1}^{(0)}$. Здесь одно семейство степенных асимптотик

$$
\mathscr{F}_{1}^{(0)} 3=\{y=c x, c \in \mathbb{C}, c \neq 0, \omega=-1, a=-1\}
$$

Если $a<-1$, то оба корня (2.7) комплексные с вешественной частью $r=1$. Поскольку $-(1,1) \in \mathbf{U}_{1}^{(0)}$, то имеем два семейства подходяших степенных асимптотик

$$
\begin{aligned}
& \mathscr{F}_{1}^{(0)} 4=\left\{y=c x^{\rho_{1}}, \omega=-1, a \in(-\infty,-1)\right\}, \\
& \mathscr{F}_{1}^{(0)} 5=\left\{y=c x^{\rho_{2}}, \omega=-1, a \in(-\infty,-1)\right\},
\end{aligned}
$$

где $c \neq 0$ - любая постоянная из $\mathbb{C}$.

Согласно п. 1.4 найдем теперь критические числа этих степенных решений. Для этого выгислим первую вариацию

$$
\frac{\delta \hat{f}_{1}^{(0)}}{\delta y}=2 x^{2} y^{\prime} \frac{d}{d x}-2 x^{2} y^{\prime \prime}-2 x^{2} y \frac{d^{2}}{d x^{2}}+2 a y .
$$

По теореме 1.2 на решении $y=c x^{\rho}$ получаем оператор

$$
\mathscr{L}(x)=2 c x^{\rho}\left[\rho x \frac{d}{d x}-\rho(\rho-1)-x^{2} \frac{d^{2}}{d x^{2}}+a\right] .
$$

Применяя оператор $\mathscr{L}(x)$ к $x^{k}$ и сокрашая результат на $x^{\rho+k}$, получаем характеристический многочлен

$$
\nu(k)=2 c\left[\rho k-\rho^{2}+\rho-k(k-1)+a\right] .
$$

Согласно (2.6) $a=\rho^{2}-2 \rho$, поэтому

$$
\nu(k)=2 c[\rho k-k(k-1)-\rho]=2 c(k-1)(\rho-k) .
$$


Следовательно, асимптотика $y=c x^{\rho}$ имеет два собственных числа $k_{1}=1$ и $k_{2}=\rho$. Очевидно, что число $k_{2}=\rho$ не является критическим. Число $k_{1}=1$ является критическим только в случае $k_{1}>\operatorname{Re} \rho$, т.е. только на семействе $\mathscr{F}_{1}^{(0)} 2$.

Итак, вершине $\Gamma_{1}^{(0)}=Q_{1}$ соответствуют пять семейств подходяших степенных решений $\mathscr{F}_{1}^{(0)} 1, \ldots, \mathscr{F}_{1}^{(0)} 5$ и только одно из них, $\mathscr{F}_{1}^{(0)} 2$, имеет критическое значение $k=1$.

Вершинам $\Gamma_{2}^{(0)}$ и $\Gamma_{3}^{(0)}$ соответствуют алгебраические укорочения $\hat{f}_{2}^{(0)}(x)=x^{2} y^{2}$ и $\hat{f}_{3}^{(0)}(x)=-x^{4}$. Согласно замечанию 1.1 соответствуюшие укороченные уравнения не имеют подходяших степенных решений.

2.3. Ребро $\Gamma_{1}^{(1)}$. Ему соответствует укороченное уравнение

$$
\hat{f}_{1}^{(1)}(X) \stackrel{\text { def }}{=} x^{2} y^{\prime 2}-2 x^{2} y y^{\prime \prime}+a y^{2}-x^{4}=0 \text {. }
$$

Поскольку внешней нормалью к ребру $\Gamma_{1}^{(1)}$ является вектор $N_{1}=-(1,2)$, то здесь $x \rightarrow 0, \omega=-1$ и у уравнения (2.14) ишем степенные решения вида $y=c x^{2}$. Подставляя это выражение в (2.14), сокрашая на $x^{4}$ и приводя подобные члены, получаем определяющее уравнение для коэффициента $c$

$$
a c^{2}-1=0 .
$$

Следовательно,

$$
c^{2}=a^{-1} \quad \text { и } \quad c_{1,2}= \pm \sqrt{a^{-1}} .
$$

При $a \neq 0$ получаем два семейства степенных асимптотик

$$
\begin{aligned}
& \mathscr{F}_{1}^{(1)} 1=\left\{y=\sqrt{a^{-1}} x^{2}, \omega=-1, a \in \mathbb{R}, a \neq 0\right\}, \\
& \mathscr{F}_{1}^{(1)} 2=\left\{y=-\sqrt{a^{-1}} x^{2}, \omega=-1, a \in \mathbb{R}, a \neq 0\right\} .
\end{aligned}
$$

Поскольку для первых вариаций справедливо равенство

$$
\delta \hat{f}_{1}^{(1)}(X) / \delta y=\delta \hat{f}_{1}^{(0)}(X) / \delta y
$$

то формула (2.11) применима и здесь, а формулы (2.12) и (2.13) справедливы при $\rho=2$ и $c=c_{1,2}$. Следовательно,

$$
\nu(k)=2 c_{1,2}\left[2 k+a-2-k^{2}+k\right]=-2 c_{1,2}\left[k^{2}-3 k+2-a\right] .
$$

Собственные числа суть $k_{1,2}=(3 \pm \sqrt{1+4 a}) / 2$. При $a>0$ одно из них $k_{1}>2$ и является критическим. При $a<0$ оба собственных числа имеют $\operatorname{Re} k_{1,2}<2$ и не являются критическими.

Итак, здесь имеются два семейства степенных асимптотик (2.16). Они имеют одно критическое число $k_{1}=(3+\sqrt{1+4 a}) / 2$ при $a>0$ и не имеют критических чисел при $a<0$.

2.4. Ребро $\Gamma_{2}^{(1)}$. Ему соответствует укороченное уравнение

$$
\hat{f}_{2}^{(1)}(X) \stackrel{\text { def }}{=} x^{2} y^{\prime 2}-2 x^{2} y y^{\prime \prime}+a y^{2}+x^{2} y^{2}=0 \text {. }
$$


Поскольку к ребру $\Gamma_{2}^{(1)}$ нормальньм является вектор $N_{2}=(0,1)$, то здесь нет степенных решений вида (1.9).

2.5. Ребро $\Gamma_{3}^{(1)}$. Ему соответствует укороченное уравнение

$$
\hat{f}_{3}^{(1)}(X) \stackrel{\text { def }}{=} x^{2} y^{2}-x^{4}=0
$$

и нормальный конус $\mathbf{U}_{3}^{(1)}=\left\{P=\lambda N_{3}=\lambda(1,1), \lambda>0\right\}$. Поскольку $N_{3}=(1,1)$, то $r=1, \omega=1, x \rightarrow \infty$ и решение укороченного уравнения ишем в виде $y=c x$. Подставляя это выражение в $(2.18)$ и сокрашая результат на $x^{4}$, для $c$ получаем определяющее уравнение $c^{2}=1$. Следовательно,

$$
c_{1,2}= \pm 1
$$

т.е. имеются два семейства степенных решений

$$
\mathscr{F}_{3}^{(1)} 1=\{y=x, a \in \mathbb{R}\}, \quad \mathscr{F}_{3}^{(1)} 2=\{y=-x, a \in \mathbb{R}\}
$$

Поскольку укороченное уравнение (2.18) алгебраическое и корни $c= \pm 1$ уравнения (1.11) простые, то согласно замечанию 1.2 решения (2.19) не имеют собственных (а следовательно, и критических) чисел и для них $\nu(k) \equiv$ const $\neq 0$.

Итак, ребру $\Gamma_{3}^{(1)}$ соответствуют два семейства степенных асимптотик (2.19), которые не имеют критических значений.

2.6. Сводка результатов. В этом параграфе найдены следующие степенные асимптотики решений уравнения (2.1).

I. Два двупараметрических (по с и $a$ ) семейства $\mathscr{F}_{1}^{(0)} 1$ и $\mathscr{F}_{1}^{(0)} 2$ из (2.8).

II. Одно однопараметрическое (по $c$ ) семейство $\mathscr{F}_{1}^{(0)} 3$ из (2.9).

III. Два двупараметрических (по с и $a$ ) семейства $\mathscr{F}_{1}^{(0)} 4$ и $\mathscr{F}_{1}^{(0)} 5$ из (2.10).

IV. Два однопараметрических (по а) семейства $\mathscr{F}_{1}^{(1)} 1$ и $\mathscr{F}_{1}^{(1)} 2$ из (2.16).

V. Два однопараметрических (по $a$ ) семейства $\mathscr{F}_{3}^{(1)} 1$ и $\mathscr{F}_{3}^{(1)} 2$ из (2.19).

\section{§ 3. Степенно-логарифмические разложения решений}

3.1. Постановка задачи. Если для уравнения (1.15) с $\nu(k) \not \equiv 0$ искать решения в виде степенного ряда

$$
z=\sum c_{k} x^{k}, \quad \omega k<\omega r
$$

где $c_{k}=$ const $\in \mathbb{C}$, то согласно [4], [5] такое разложение решений существует только при определенных условиях. При этом основное условие - это отсутствие критических значений. Если же не накладьвать этих условий, то получаются разложения вида (3.1), где $c_{k}$ суть многочлены от $\ln x$. Но такого же вида разложения решений получаются и у уравнений более обшего вида, чем (1.15). Поэтому расширим класс уравнений.

Сначала дополним определения $\S 1$. Дифференциально-логарифмическим (ДЛ-) мономом назьвается произведение дифференциального монома $a(x, z)$ на многочлен $\alpha(\ln x)$ от $\ln x$. Векторньй показатель степени $Q=\left(q_{1}, q_{2}\right)$ ДЛ-монома

$$
\alpha(\ln x) a(x, z)
$$


равен показателю степени дифференциального монома $a(x, z)$. Сначала рассмотрим случай, когда в сомножителе

$$
c x^{r_{1}} z^{r_{2}}
$$

дифференциального монома $a(x, z)$ показатель $r_{1} \in \mathbb{R}$, а в конце параграфа - случай $r_{1} \in \mathbb{C}$.

Дифференциально-логарифмической (ДЛ-) суммой $f(x, z)$ назьвается (конечная или бесконечная) сумма ДЛ-мономов. Если это бесконечная сумма, то будем предполагать, что в ней (а) конечно число ДЛ-мономов с одним показателем $Q$ и (б) показатели $Q$ ДЛ-мономов не имеют точек накопления в $\mathbb{R}^{2}$.

ДЛ-сумме $f(x, z)$ соответствует множество $\mathbf{S}(f)$ векторных показателей степеней ее ДЛ-мономов, называемое носителем суммы $f(x, z)$, и многоугольник $\Gamma(f)$, являющийся замыканием вьпуклой оболочки носителя $\mathbf{S}(f)$.

Рассмотрим уравнение

$$
\tilde{f}(x, z) \stackrel{\text { def }}{=} \mathscr{L}(x) z+h(x, z)=0
$$

где $\tilde{f}(x, z)$ - ДЛ-сумма, у всех ДЛ-мономов которой в сомножителе $(3.2)$ число $r_{2}$ целое неотрицательное, а $\mathscr{L}(x)$ - линейньй дифференщиальньй оператор.

ЗАДАчА 2. Для решений уравнения (3.3) найти разложения

$$
z=\sum \beta_{k}(\ln x) x^{k}
$$

где $\beta_{k}$ суть многочлены от $\ln x$ с комплексньми коэффищиентами и показатели $k$ или $\operatorname{Re} k$ лежат в конусе задачи (1.14), если он есть.

3.2. Носитель разложения решения. Для определенности на слагаемые в уравнении (3.3) наложим следуюшие условия.

УСловиЕ 3.1. Точка $(v, 1)$ является вершиной многоугольника $\Gamma(\tilde{f})$. В сумме $\tilde{f}(x, z)$ ей соответствует слагаемое $\mathscr{L}(x) z$ и только оно. ДЛ-сумма $\mathscr{L}(x) z$ не содержит логарифмов, т.е. является дифференциальной суммой.

Если уравнение (3.3) получено из (1.4) и $\mathscr{L} \not \equiv 0$, то это условие выполняется автоматически. Если это условие вьполнено, то дифференщиальная сумма $\mathscr{L}(x) z$ имеет характеристический многочлен $(1.17)$ и $\nu(k) \not \equiv 0$.

Сдвинем параллельно носитель $\mathbf{S}(\tilde{f})$ на вектор $(-v,-1)$. Тогда вершина $(v, 1)$, соответствуюшая члену $\mathscr{L}(x) z$, перейдет в начало координат. Пусть задано такое число $r$, что для всякой точки $Q^{\prime} \in \mathbf{S}^{\prime} \stackrel{\text { def }}{=} \mathbf{S}(\tilde{f})-(v, 1)$ скалярное произведение $\left\langle\omega R, Q^{\prime}\right\rangle \geqslant 0$, где $R=(1, r)$. Пусть вешественные числа $k_{1}, \ldots, k_{s}$ удовлетворяют неравенству $k \omega<r \omega$ из (1.14). Пусть $\mathbf{S}_{+}^{\prime}\left(k_{1}, \ldots, k_{s}\right)$ - множество конечных сумм векторов $Q^{\prime} \in \mathbf{S}^{\prime}$ и векторов $\left(k_{1},-1\right), \ldots,\left(k_{s},-1\right)$. Обозначим

$$
\mathbf{K}\left(k_{1}, \ldots, k_{s}\right)=\mathbf{S}_{+}^{\prime}\left(k_{1}, \ldots, k_{s}\right) \cap\left\{q_{2}=-1\right\} .
$$

ПРЕДЛОЖЕНИЕ 3.1. Множество $\mathbf{K}\left(k_{1}, \ldots, k_{s}\right)$ не имеет точек накопления в $\mathbb{R}$, если носитель $\mathbf{S}(\tilde{f})$ не имеет точек накопления в $\mathbb{R}^{2}$. 
ПРЕДЛОЖЕНИЕ 3.2. Пусть $\Gamma_{j}^{(0)}$ - такая вериина многоугольника $\Gamma(f)$ уравнения (1.4), что соответствующее укороченное уравнение (1.9) имеет решение (1.7) $c \omega(1, r) \in \mathbf{U}_{j}^{(0)}$ и все точки сдвинутого носителя $\mathbf{S}(f)-\Gamma_{j}^{(0)}$ представляются в виде $\sum_{i=1}^{n} l_{i} M_{i}$, где челье $l_{i} \geqslant 0$, а $M_{i} \in \mathbb{R}^{2}$ - некоторие векторы. Тогда для множества $\mathbf{K}$ уравнения (3.3) справедливо включение

$$
\mathbf{K} \subset\left\{k=r+\sum_{i=1}^{n} l_{i} r_{i}, \text { uелье } l_{i} \geqslant 0, \sum_{i=1}^{n} l_{i}>0\right\},
$$

əде $r_{i}=\left\langle(1, r), M_{i}\right\rangle, i=1, \ldots, n$.

ПРЕДЛОЖЕНИЕ 3.3. Если множество

$$
\mathbf{K}=\left\{k=r+\sum_{i=1}^{n} l_{i} r_{i}, \text { иельве } l_{i} \geqslant 0, \sum_{i=1}^{n} l_{i}>0\right\}
$$

то множество (3.4) имеет вид

$$
\begin{aligned}
& \mathbf{K}\left(k_{1}, \ldots, k_{s}\right)=\left\{k=r+\sum_{i=1}^{n} l_{i} r_{i}+\sum_{j=1}^{s} m_{j}\left(k_{j}-r\right),\right. \\
&\text { чельие } \left.l_{i}, m_{j} \geqslant 0, \sum_{i=1}^{n} l_{i}+\sum_{j=1}^{s} m_{j}>0\right\} .
\end{aligned}
$$

\section{3. Вычисление разложений.}

Теорема 3.1. Если уравнение (3.3) удовлетворяет условию 3.1 , то оно имеет формальное решение

$$
z=z^{*}(x) \stackrel{\text { def }}{=} \sum \beta_{k}(\ln x) x^{k}, \quad k \in \mathbf{K}\left(k_{1}, \ldots, k_{s}\right),
$$

где $\beta_{k}(\ln x)$ суть многочлены от $\ln x$ и $k_{1}, \ldots, k_{s}$ - критические числа укороченного решения (1.7).

Действительно, двигаясь по точкам $k$ множества (3.4) в направлении возрастания $-\omega(k-r)$, для каждого коэффищиента $\beta_{k}$ из (3.5) получаем линейное уравнение

$$
\mathscr{L}(x) \beta_{k} x^{k}+\theta_{k} x^{k+v}=0,
$$

где $\theta_{k}-$ многочлен от коэффициентов $\beta_{j}$ и их производных с $-\omega(j-r)<-\omega(k-r)$, т.е. $-\omega j<-\omega k$. Кроме того, коэффициент $\theta_{k}$ зависит от коэффициентов суммы $h$ в (3.3). На самом деле, $\theta_{k}-$ это коэффициент при $x^{k+v}$ в сумме

$$
h\left(x, \sum_{-\omega r<-\omega j<-\omega k} \beta_{j} x^{j}\right) .
$$

Пусть утверждение теоремы справедливо для всех $\beta_{j} \mathrm{c}-\omega j<-\omega k$. Тогда $\theta_{k}$ является многочленом от $\xi \stackrel{\text { def }}{=} \ln x$. 
ЛЕмма 3.1. Уравнение (3.6) әквивалентно линейному дифференциальному уравнению

$$
\mathscr{N}_{k}(\xi) \beta_{k}(\xi)+\theta_{k}(\xi) \stackrel{\text { def }}{=} \sum \frac{1}{m !} \nu^{(m)}(k) \beta_{k}^{(m)}(\xi)+\theta_{k}(\xi)=0
$$

где $\nu^{(m)}(k)=\left.\frac{d^{m} \nu(q)}{d q^{m}}\right|_{q=k}, \beta_{k}^{(m)}(\xi)=\frac{d^{m} \beta_{k}(\xi)}{d \xi^{m}}, m=0,1,2, \ldots$

Пусть $\mu(k)$ - наименьшее значение $m$, для которого $\nu^{(m)}(k) \neq 0$, и $\lambda(k)$ - степень многочлена $\theta_{k}(\xi) ;$ при этом $\lambda(k)=-1$, если $\theta_{k} \equiv 0$.

Лемма 3.2. Пусть $\theta_{k}(\xi)$ - многочлен степени $\lambda(k)$, тогда уравнение (3.7) имеет решение $\beta_{k}(\xi)$, являюшееся многочленом степени $\mu(k)+\lambda(k)$ и содерэсащее $\mu(k)$ произвольных коэффичиентов.

Действительно, оператор $\mathscr{N}_{k}(\xi)$ в (3.7) имеет вид

$$
\mathscr{N}_{k}^{(\mu)}(\xi)+\mathscr{N}_{k}^{(\mu+1)}(\xi)+\cdots
$$

где $\mathscr{N}_{k}^{(\mu)}$ означает оператор порядка $\mu$. Если разложить многочлены $\beta_{k}(\xi)$ и $\theta_{k}(\xi)$ по степеням $\xi$ в виде

$$
\beta_{k}=\sum_{l=0}^{\mu+\lambda} \beta_{k l} \xi^{l}, \quad \theta_{k}=\sum_{l=0}^{\lambda} \theta_{k l} \xi^{l}
$$

и в обеих частях уравнения (3.7) приравнять члены с одинаковыми степенями $\xi$, то получается неоднородная линейная система $\lambda+1$ уравнений для $\lambda+\mu+1$ коэффициентов $\beta_{k l}$. Будем вычислять эти коэффициенты, начиная со старшей степени $l=\mu(k)+\lambda(k)$. Для этого коэффициента получается уравнение

$$
\mathscr{N}_{k}^{(\mu)} \beta_{k, \mu+\lambda} \xi^{\mu+\lambda}+\theta_{k, \lambda} \xi^{\lambda}=0
$$

т.е. согласно (3.7)

$$
\frac{1}{\mu !} \nu^{(\mu)}(k) \frac{(\mu+\lambda) !}{\lambda !} \beta_{k, \mu+\lambda}+\theta_{k, \lambda}=0
$$

По определению $\mu$ коэффициент $\nu^{(\mu)}(k) \neq 0$, поэтому это уравнение имеет однозначное решение. Для коэффициента $\beta_{k, \mu+\lambda-1}$ получается аналогичное уравнение, только правая часть зависит от $\theta_{k, \lambda-1}$ и $\beta_{k, \mu+\lambda}$ и т. д. Наконец, согласно $(3.7)$ для $l<\mu(k)$ коэффициент $\beta_{k, l}$ отсутствует в указанной линейной системе и может быть взят любьмм.

ЗАмЕчАнИЕ 3.1. Зная оператор $\mathscr{L}(x)$ и носитель $\mathbf{K}\left(k_{1}, \ldots, k_{s}\right)$ разложения $(3.5)$, можно вычислять его коэффициенты прямо по исходному уравнению $f(x, y)=0$, ибо коэффициент при $x^{k}$ в сумме $\left(3.6^{\prime}\right)$ совпадает с коэффициентом при $x^{k+v}$ в сумме

$$
f\left(x, c_{r} x^{r}+\sum_{-\omega r<-\omega j<-\omega k} \beta_{j} x^{j}\right) .
$$


Следовательно, вычислив начальный отрезок разложения

$$
y=c_{r} x^{r}+\sum \beta_{j} x^{j}
$$

и подставив его в $f(x, y)$, получаем функщию $\theta_{k}$ в уравнении (3.7). В сложных случаях это уравнение можно решать так, как описано в пояснении к лемме 3.2 .

Семейство разложений решений $(3.5)$, соответствуюшее семейству $\mathscr{F}_{j}^{(d)} l$ степенных асимптотик решений $(1.7)$, будем обозначать $\mathscr{G}_{j}^{(d)} l$. Как правило, удается вычислить не все разложение (3.5), а только его начальньй отрезок. При этом желательно, чтобы этот отрезок содержал все показатели $k$, соответствуюшие критическим значениям $k_{1}, \ldots, k_{s}$. Тогда он содержит все произвольные постоянные семейства $\mathscr{G}_{j}^{(d)} k$. Семейство такого отрезка разложения (3.5) будем обозначать $\tilde{\mathscr{G}}_{j}^{(d)} k$.

ЗАмЕчАниЕ 3.2. Если критические числа $k_{1}, \ldots, k_{s}$ таковы, что числа $k_{t+1}, \ldots, k_{s}$ не лежат в множестве $\mathbf{K}\left(k_{1}, \ldots, k_{t}\right)$, то уравнение (3.3) имеет решение вида $(3.5)$, носитель которого лежит в множестве $\mathbf{K}\left(k_{1}, \ldots, k_{t}\right)$.

3.4. Степени логарифмов в разложении. Очевидно, что степень ord $\beta_{k}(\xi)$ многочленов $\beta_{k}$ растет вместе с ростом $-\omega(k-r)$. Оценим ее сверху для случая, когда уравнение (3.3) не содержит логарифмов. Положим $\varkappa(j)=\mu(j) /|j-r|$ и $q^{*}=\max q_{2}$ по $\left(q_{1}, q_{2}\right) \in \mathbf{S}(\tilde{f})$.

ТЕОРема 3.2. Если в уравнении (3.3) нет логарифмов, то в ситуации замечания 3.2 в решении (3.5)

$$
\operatorname{ord} \beta_{k} \leqslant q^{*}|k-r| \sum_{0<-\omega(j-r)<-\omega(k-r)} \varkappa(j),
$$

где $j, k \in \mathbf{K}\left(k_{1}, \ldots, k_{t}\right)$.

Говорят, что для критического числа $k_{i}$ выполнено условие совместности, если для $k=k_{i}$ в уравнении $(3.6) \theta_{k} \equiv 0$.

ЗАмечАниЕ 3.3. В ситуации теоремы 3.2 разложение (3.5) может содержать логарифм $\ln x$ только в двух случаях: (а) если в множестве $\mathbf{K}\left(k_{1}, \ldots, k_{i-1}, k_{i}, \ldots, k_{t}\right)$ лежит критическое число $k_{i}$, для которого не выполнено условие совместности; (б) если среди $k_{1}, \ldots, k_{t}$ имеется кратное критическое число. Это согласуется с результатами из $[4],[5]$.

3.5. Решетка носителя разложения. Дискретное множество $\mathbf{Z}$ в $\mathbb{R}^{n}$ назьвается решеткой, если оно замкнуто относительно векторных сложения и вычитания. Векторы $B_{1}, \ldots, B_{n}$ образуют базис решетки $\mathbf{Z}$, если всякая точка $Q \in \mathbf{Z}$ может быть представлена в виде

$$
Q=m_{1} B_{1}+\cdots+m_{n} B_{n}, \text { где все } m_{i} \in \mathbb{Z} \text {. }
$$


Tеорема 3.3 [4], [5]. Если множество $\mathbf{S}^{\prime} \stackrel{\text { def }}{=} \mathbf{S}(f)-(v, 1)$ и точки $\left(k_{1},-1\right), \ldots$, $\left(k_{s},-1\right)$ лежсат в некоторой решетке $\mathbf{Z}$, то множество

$$
\mathbf{K}\left(k_{1}, \ldots, k_{s}\right) \subset \mathbf{Z} \cap\left\{q_{2}=-1\right\} .
$$

3.6. Комплексные показатели. Сначала рассмотрим случай, когда в уравнении (3.3) у всех ДЛ-мономов в множителе (3.2) показатель $r_{1}$ вешественньй, но среди собственных чисел $k_{i}$ имеются комплексные, у которых вешественная часть Re $k_{i}$ лежит в конусе задачи (1.14). Так что теперь $k_{1}, \ldots, k_{s}$ - это все критические значения оператора $\mathscr{L}(x)$, включая комплексные, т.е. $\omega$ Re $k_{i}<\omega r$. В этом случае множество $\mathbf{S}_{+}^{\prime}\left(k_{1}, \ldots, k_{s}\right)$ содержит точки $Q=\left(q_{1}, q_{2}\right)$, у которых $q_{1} \in \mathbb{C}, q_{2} \in \mathbb{Z}$, а $\mathbf{K}$ - это множество на комплексной плоскости $q_{1} \in \mathbb{C}$. Тогда теорема 3.1 остается справедливой, только в разложении (3.5) показатели $k$ частично упорядочены по росту $-\omega(\operatorname{Re} k-r)$. Все дальнейшие утверждения пп. 3.2-3.5 также остаются справедливыми.

Если же в уравнении (3.3) имеются $x^{r_{1}}$ с комплексным показателем $r_{1}$, то носитель $\mathbf{S}(\tilde{f})$ лежит в прямой сумме $\mathbb{C} \oplus \mathbb{R}$. Но при построении многоугольника $\Gamma(\tilde{f})$ учитываются только $\operatorname{Re} q_{1}$, т.е. $\operatorname{Re} \mathbf{S}(\tilde{f})$. Все дальнейшие конструкции и результаты сохраняются. Разложения решений (3.1) с комплексными показателями $k$ рассматривались в $[37]$

3.7. Существование решений. Если для уравнения (3.3) разложение (3.5) сходится при достаточно малых $|x|^{-\omega}$, то этому разложению соответствует решение уравнения (3.3). Для дифференциальной суммы $\tilde{f}$ в (3.3) условия сходимости степенного разложения (3.5) приведены в [3]-[5]. Максимальньй порядок производной в дифференциально-логарифмической сумме $f(x, z)$ назовем ее порядком дифференцирования и обозначим $\pi(f)$.

ТЕОРема 3.4. Для дифференциальной суммы $\tilde{f}$ уравнения (3.3) степенное разложсение его решения (3.5) сходится для достаточно малых $|x|^{-\omega} \neq 0$, если

$$
\pi(\mathscr{L} z)=\pi(\tilde{f})
$$

Очевидно, что $\pi(\tilde{f})=\pi(f)$, но, вообще говоря, $\pi(\mathscr{L} z) \leqslant \pi(\hat{f})$, хотя строгое неравенство здесь имеется только в вырожденных случаях.

Если дифференциальное уравнение (3.3) и разложение (3.5) вешественны, то у уравнения (3.3) всегда существует такое вешественное решение, для которого ряд (3.5) является асимптотическим разложением. Вообще говоря, такое решение не единственно. Даже разложение с комплексными показателями может быть вещественньм для вещественного аргумента, если оно переходит в себя при комплексном сопряжении аргумента.

ГИПОТЕЗА 3.1. В ситуации теоремы 3.1 для любого достаточно узкого сектора комплексной плоскости $x^{-\omega}$ уравнение $(3.3)$ имеет решение $z(x)$, для которого ряд (3.5) является асимптотическим разложением. 


\section{§4. Примеры вычисления разложений}

Продолжим рассмотрение уравнения (2.1), начатое в $§ 2$, и покажем, какие разложения (3.5) получаются из результатов $\S 3$ для различных семейств $\mathscr{F}_{j}^{(d)} k$ степенных асимптотик, найденных в $\S 2$.

4.1. Семейство $\mathscr{F}_{1}^{(0)} 1$ из (2.8). Здесь $r=r_{1}=1+\sqrt{1+a} \in(1,2)$ и нет критических чисел. Для

$$
\tilde{f}(x, z) \stackrel{\text { def }}{=} f\left(x, c x^{r}+z\right)
$$

носитель $\mathbf{S}(\tilde{f})$ содержит кроме точек (2.2) еще три точки

$$
Q_{4}=(r, 1), \quad Q_{5}=(r+2,1), \quad Q_{6}=(2 r+2,0)
$$

(рис. 3). При этом точка $Q_{4}$ является вершиной $(v, 1)$, т.е. $v=r$. После сдвига носителя $\mathbf{S}(f)$ на вектор $(-r,-1)$ получаем 6 точек $Q_{i}^{\prime}=Q_{i}-Q_{4}$ :

$$
\begin{gathered}
Q_{1}^{\prime}=(-r, 1), \quad Q_{2}^{\prime}=(2-r, 1), \quad Q_{3}^{\prime}=(4-r,-1), \\
Q_{4}^{\prime}=0, \quad Q_{5}^{\prime}=(2,0), \quad Q_{6}^{\prime}=(r+2,-1),
\end{gathered}
$$

показанных на рис. 4 . Множество $\mathbf{S}_{+}^{\prime}$ конечных сумм векторов $Q_{1}^{\prime}, \ldots, Q_{6}^{\prime}$ расположено в секторе, ограниченном лучами, натянутыми на $Q_{1}^{\prime}$ и $Q_{3}^{\prime}$ (рис. 4 ). При этом

$$
Q_{2}^{\prime}=2 Q_{1}^{\prime}+Q_{6}^{\prime}, \quad Q_{5}^{\prime}=Q_{1}^{\prime}+Q_{6}^{\prime}
$$

Поэтому множество $\mathbf{S}_{+}^{\prime}$ порождается векторами $Q_{1}^{\prime}, Q_{3}^{\prime}, Q_{6}^{\prime}$ и состоит из точек

$$
Q=j Q_{1}^{\prime}+l Q_{3}^{\prime}+m Q_{6}^{\prime} \text {, где целые } j, l, m \geqslant 0 .
$$

Согласно (4.3) для этих точек $Q=\left(q_{1}, q_{2}\right)$ имеем $q_{1}=-j r+l(4-r)+m(r+2)$, $q_{2}=j-l-m$. На прямой $q_{2}=-1$ имеем $j-l-m=-1$, т.е. $j=l+m-1$, где $l+m>0$. Поэтому $q_{1}=-(l+m-1) r+l(4-r)+m(r+2)=r+2 l(2-r)+2 m$. Этот же результат следует из предложения 3.2 , ибо для $\Gamma_{j}^{(0)}$ имеем $n=2, M_{1}=2(2,-1)$ и $M_{2}=2(1,0)$, т.е. $r_{1}=2(2-r)$ и $r_{2}=2$. Следовательно, множество (3.4) есть

$$
\mathbf{K}=\{k=r+2 l(2-r)+2 m ; \text { целые } l, m \geqslant 0, l+m>0\} .
$$

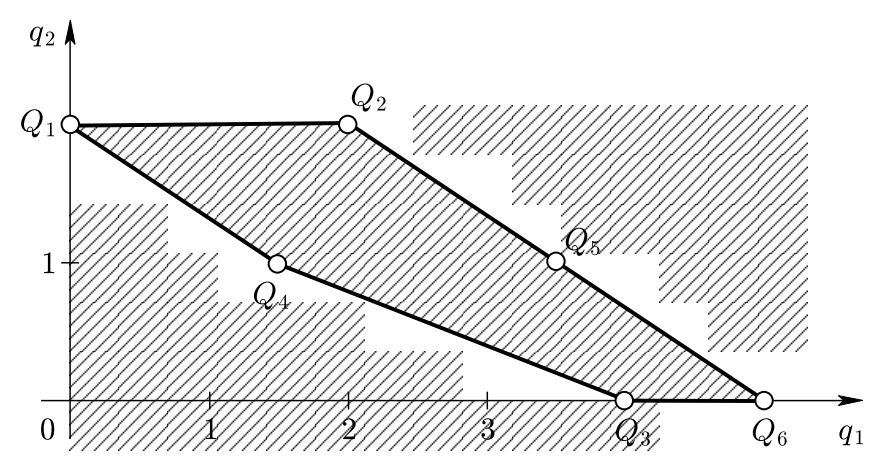

Рис. 3. 


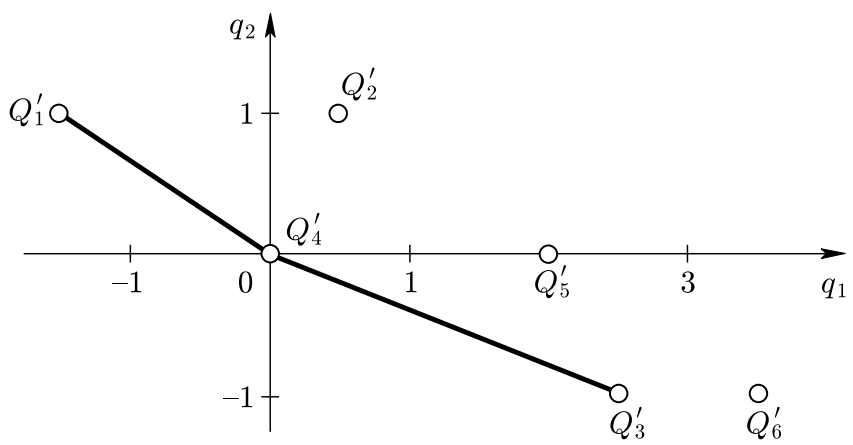

Рис. 4.

Согласно теореме 3.1 и замечанию 3.2 в этом случае семейство $\mathscr{G}_{1}^{(0)} 1$ состоит из разложений (3.1) с постоянными однозначно определенными коэффициентами $c_{k}$, где показатели $k$ пробегают множество (4.6). Если число $r$ рационально со знаменателем $t$, то множество $\mathbf{S}_{+}^{\prime}$ содержится в решетке $\mathbf{Z}$ с базисньми векторами $B_{1}=\left(t^{-1}, 1\right)$, $B_{2}=\left(2 t^{-1}, 0\right)$. По теореме 3.3 множество $\mathbf{K}$ содержится в множестве $\{k=4-r+$ $\left.2 l t^{-1}, l=0,1,2, \ldots\right\}$.

4.2. Семейство $\mathscr{F}_{1}^{(0)} 2$ из (2.8). Для него $r=r_{2}=1-\sqrt{1+a}<1$ и имеется одно критическое число $k_{1}=1$. Здесь равенства (4.2)-(4.4) остаются, но расположение точек (4.3) другое (рис. 5). Множество $\mathbf{S}_{+}^{\prime}$ расположено в секторе, ограниченном лучами, натянутыми на векторы $Q_{1}^{\prime}$ и $Q_{6}^{\prime}$, оно опять порождается векторами $Q_{1}^{\prime}, Q_{3}^{\prime}$, $Q_{6}^{\prime}$ и состоит из точек (4.5). Поэтому множество К есть (4.6). Критическое значение $k_{1}=1$ попадает в это множество только при $l=0$ и $r=1-2 m$, т.е. при

$$
a=(2 m-1)(2 m+1), \quad m=1,2, \ldots
$$

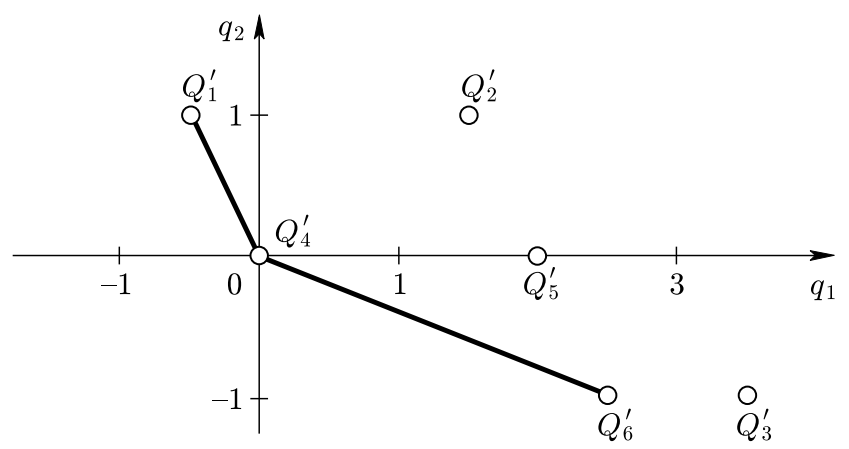

Рис. 5.

Если равенства (4.7) не вьполнены, то множество $\mathbf{S}_{+}^{\prime}\left(k_{1}\right)=\mathbf{S}_{+}^{\prime}(1)$ порождается векторами $Q_{1}^{\prime}, Q_{3}^{\prime}, Q_{6}^{\prime}$ и состоит из точек

$$
Q=j Q_{1}^{\prime}+l Q_{3}^{\prime}+m Q_{6}^{\prime}+n(1,-1), \text { где целые } j, l, m, n \geqslant 0 .
$$


Согласно (4.3) для этих точек $Q=\left(q_{1}, q_{2}\right)$ имеем $q_{1}=-j r+l(4-r)+m(r+2)+n$, $q_{2}=j-l-m-n$. На прямой $q_{2}=-1$ имеем $j-l-m-n=-1$, т.е. $j=l+m+n-1$, где $l+m+n>0$. Следовательно,

$$
\mathbf{K}(1)=\{k=r+2 l(2-r)+2 m+n(1-r) ; \text { целые } l, m, n \geqslant 0, l+m+n>0\} .
$$

Этот же результат следует из предложения 3.3 и формулы (4.6), ибо здесь $s=1$, $k_{1}-r=1-r$. В этом случае семейство $\mathscr{G}_{1}^{(0)} 2$ состоит из разложений $(3.1)$ с произвольным постоянньм коэффициентом $c_{1}$ и с однозначно определенными постоянными коэффициентами $c_{k}$ для $k \neq 1$, где показатели $k$ пробегают множество (4.8).

Если вьполнено одно из равенств (4.7), то множество К(1) совпадает с множеством (4.6) и в разложении решения (3.5) появляются логарифмы для $\beta_{k} \mathrm{c} k \geqslant 1$. Рассмотрим подробно случай (4.7) с $m=1$. Тогда $a=3, r=-1$, множество (4.6) - это множество нечетных чисел $\{k=1,3,5, \ldots\}$. Будем искать первьй член разложения (3.5) с $k=1$, т.е.

$$
y=c x^{-1}+\beta x+\cdots .
$$

Тогда $y^{\prime}=-c x^{-2}+\beta+\beta^{\prime} x+\cdots, y^{\prime \prime}=2 c x^{-3}+2 \beta^{\prime}+\beta^{\prime \prime} x+\cdots$. Подставляя эти выражения в уравнение $(2.1)$ и выписьвая члены с $x^{-2}$ и $x^{0}$, получаем

$$
\begin{aligned}
& x^{2}\left(-c x^{-2}+\beta^{\prime} x+\beta\right)^{2}-2 x^{2}\left(c x^{-1}+\beta x\right)\left(2 c x^{-3}+\beta^{\prime \prime} x+2 \beta^{\prime}\right) \\
& +3\left(c x^{-1}+\beta x\right)^{2}+x^{2}\left(c x^{-1}+\beta x\right)^{2}+\cdots \\
& \quad=-2 c\left(\beta^{\prime} x+\beta\right)-4 c \beta-2 c\left(\beta^{\prime \prime} x^{2}+2 \beta^{\prime} x\right)+6 c \beta+c^{2}+\cdots
\end{aligned}
$$

Приравнивая к нулю вьписанную сумму членов нулевой степени по $x$ и сокращая ее на $-c$, получаем уравнение

$$
2 \beta^{\prime \prime} x^{2}+6 \beta^{\prime} x-c=0 .
$$

Положим $\xi=\ln x$ и будем точкой обозначать дифференцирование по $\xi$. Тогда

$$
\beta^{\prime}=\dot{\beta} x^{-1}, \quad \beta^{\prime \prime}=(\ddot{\beta}-\dot{\beta}) x^{-2},
$$

и уравнение $(4.9)$ принимает вид $2(\ddot{\beta}-\dot{\beta})+6 \dot{\beta}=c$, т.е.

$$
2 \ddot{\beta}+4 \dot{\beta}=c .
$$

Это уравнение имеет решения

$$
\beta=(c / 4) \xi+c_{1},
$$

где $c_{1}$ - произвольная постоянная. Следовательно, в этом случае разложение (1.12), (3.5) имеет вид

$$
y=c x^{-1}+(c / 4)\left(\ln x+c_{1}\right) x+\beta_{3} x^{3}+\cdots,
$$

где $c \neq 0$ и $c_{1}$ - произвольные постоянные, $\beta_{3}$ - многочлен второй степени от $\ln x$.

Теперь вычислим коэффициент $\beta(\ln x)$, используя замечание 3.1. Подставим в уравнение (2.1) $y=c x^{-1}$ и вычислим коэффициент при $x^{0}$, ибо здесь $v=1$. Получаем $\theta_{1}=c^{2}$. При $k=1$ уравнение (3.7) принимает вид

$$
\nu(1) \beta_{1}(\xi)+\nu^{\prime}(1) \dot{\beta}_{1}(\xi)+\frac{1}{2} \nu^{\prime \prime}(1) \beta_{1}(\xi)+c^{2}=0 .
$$


Согласно $\left(2.13^{\prime}\right)$ имеем $\nu(k)=2 c(k-1)(\rho-1)$. Но здесь $\rho=-1$, т.е. $\nu(k)=-2 c\left(k^{2}-1\right)$. Следовательно, $\nu^{\prime}(k)=-4 c k$ и $\nu^{\prime \prime}(k)=-4 c$. Поэтому $\nu(1)=0, \nu^{\prime}(1)=-4 c, \nu^{\prime \prime}(1)=$ $-4 c$ и уравнение $\left(4.10^{\prime}\right)$ принимает вид $-4 c \dot{\beta}_{1}-2 c \ddot{\beta}_{1}+c^{2}=0$, аналогичный $\left(4.9^{\prime \prime}\right)$. Более того, в обозначениях леммы 3.2 здесь $\lambda(1)=0, \mu(1)=1$ и $\lambda+\mu=1$. По лемме 3.2 многочлен $\beta(\xi)$ имеет первую степень, т.е. $\ddot{\beta}=0$.

4.3. Семейство $\mathscr{F}_{1}^{(0)} 3$ из (2.9). Для него множество $\mathbf{S}^{\prime}$ лежит в решетке $\mathbf{Z}$ с базисом $B_{1}=(2,0), B_{2}=(1,-1)$. Поэтому множество $\mathbf{K}$ состоит из нечетных чисел $\{k=3,5, \ldots\}$. Разложение (1.12), (3.5) имеет вид

$$
y=c x+x \sum_{k=1}^{\infty} c_{2 k} x^{2 k}
$$

где $c \neq 0$ - произвольная постоянная и все $c_{2 k}$ однозначно определены. Это семейство $\varphi_{1}^{(0)} 3$.

4.4. Семейство $\mathscr{F}_{1}^{(0)} 4$ из (2.10). Для него $\rho=\rho_{1}=1+\sqrt{1+a} \stackrel{\text { def }}{=} 1+i s$ и нет критических значений. Для $\tilde{f}(x, z) \stackrel{\text { def }}{=} f\left(x, c x^{\rho}+z\right)$ носитель $\mathbf{S}(\tilde{f})$ состоит из точек (2.2) и точек

$$
Q_{4}=(1+i s, 1), \quad Q_{5}=(3+i s, 1), \quad Q_{6}=(4+2 i s, 0) .
$$

Члену $\mathscr{L}(x) z$ соответствует точка $Q_{4}$. Положим $Q_{i}^{\prime}=Q_{i}-Q_{4}$. Тогда

$$
\begin{gathered}
Q_{1}^{\prime}=(-1-i s, 1), \quad Q_{2}^{\prime}=(1-i s, 1), \quad Q_{3}^{\prime}=(3-i s,-1), \\
Q_{4}^{\prime}=0, \quad Q_{5}^{\prime}=(2,0), \quad Q_{6}^{\prime}=(3+i s,-1) .
\end{gathered}
$$

На рис. 6 показана проекция этих точек на плоскость $\operatorname{Re} q_{1}, \operatorname{Im} q_{1} ;$ при этом точки $Q_{1}^{\prime}$ и $Q_{2}^{\prime}$ имеют $q_{2}=1, Q_{4}^{\prime}$ и $Q_{5}^{\prime}$ имеют $q_{2}=0$, а $Q_{3}^{\prime}$ и $Q_{6}^{\prime}$ имеют $q_{2}=-1$. Здесь справедливы равенства (4.2)-(4.4) с $\rho$ вместо $r$, и множество $\mathbf{S}_{+}^{\prime}$ порождается векторами $Q_{1}^{\prime}$, $Q_{3}^{\prime}, Q_{6}^{\prime}$. Вычисляя множество $\mathbf{K}$ так же, как для семейства $\mathscr{F}_{1}^{(0)} 1$, получаем аналог формулы (4.6), где $r$ заменено на $\rho$ :

$$
\mathbf{K}=\{k=1+i s+2 l(1-i s)+2 m ; \text { целые } l, m \geqslant 0, l+m>0\}
$$

(кружочки на рис. 7). Поэтому разложение (3.5) имеет вид

$$
z=x^{3+i s}\left(\sum_{l=0}^{\infty} c_{2 l} x^{2 l}\right)+x^{3-i s}\left(\sum_{l=0}^{\infty} c_{2 l}^{(1)} x^{2 l}\right)+x^{5-3 i s}\left(\sum_{l=0}^{\infty} c_{2 l}^{(2)} x^{2 l}\right)+\cdots
$$

где все коэффищиенты $c_{2 l}, c_{2 l}^{(1)}, c_{2 l}^{(2)}, \ldots$ постоянны и однозначно определены. Согласно $(2.10),(1.20)$ и (4.13) носитель разложения для $y$ содержит еще точку $1+i s$, показанную звездочкой на рис. 7 , и заключен в угле, выделенном пунктиром на рис. 7 . 


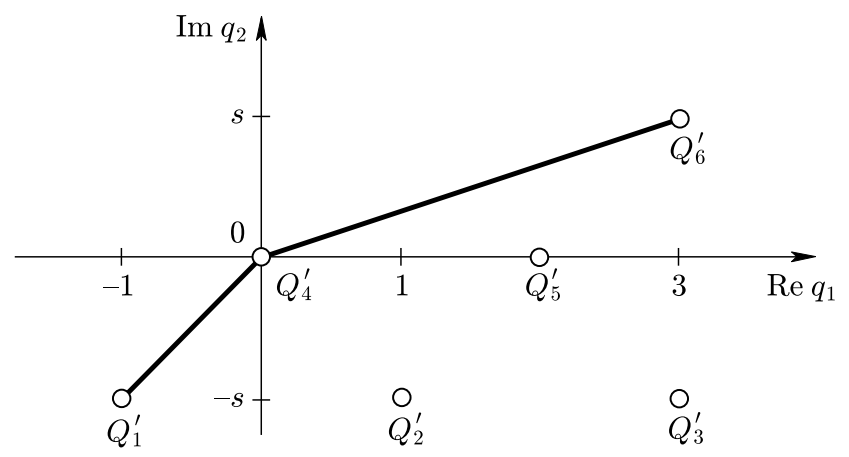

Рис. 6.

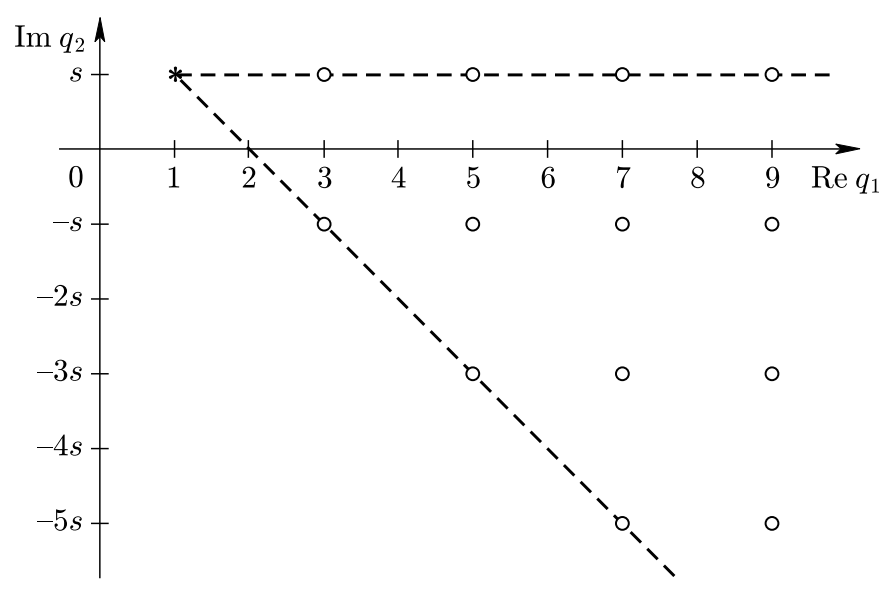

Рис. 7.

Для семейства $\mathscr{F}_{1}^{(0)} 5$ из (2.10) получается разложение вида (4.13), где $s$ надо заменить на $-s$.

4.5. Семейство $\mathscr{F}_{1}^{(1)} 1$ из (2.16). Для него $r=2$ и при $a<0$ нет критических значений, а при $a>0$ имеется одно критическое значение $k_{1}=(3+\sqrt{1+4 a}) / 2$. Носитель $\mathbf{S}(\tilde{f})$ состоит из точек

$$
Q_{1}, \quad Q_{2}, \quad Q_{4}=(2,1), \quad Q_{5}=(4,1), \quad Q_{6}=(6,0)
$$

Члену $\mathscr{L}(x) z$ соответствует вершина $Q_{4}$, поэтому $v=2$. Полагая $Q_{i}^{\prime}=Q_{i}-Q_{4}$, получаем

$$
Q_{1}^{\prime}=(-2,1), \quad Q_{2}^{\prime}=(0,1), \quad Q_{4}^{\prime}=0, \quad Q_{5}^{\prime}=(2,0), \quad Q_{6}^{\prime}=(4,-1)
$$

Все они лежат в решетке с базисом $B_{1}=(2,0) \stackrel{\text { def }}{=} 2 E_{1}, B_{2}=(0,1) \stackrel{\text { def }}{=} E_{2}$. Поэтому множество $\mathbf{K} \stackrel{\text { def }}{=} \mathbf{S}_{+}^{\prime} \cap\left\{q_{2}=-1\right\}=\{k=4+2 l, l=0,1,2, \ldots\}$. 
При $a<0$ нет критических значений. По теореме 4.1 для полусемейства $\mathscr{G}_{1}^{(1)} 1-$ разложение (1.12), (3.5) имеет вид

$$
y=c x^{2}+\sum_{k=2}^{\infty} c_{2 k} x^{2 k}
$$

где $c=\sqrt{a^{-1}}$ и все коэффициенты $c_{2 k}$ являются определенными постоянными, зависяшими от $c$. Для полусемейства $\mathscr{G}_{1}^{(1)} 2$ - разложение решений также имеет вид (4.14), только $c=-\sqrt{a^{-1}}$.

При $a>0$ имеется критическое число $k_{1}=(3+\sqrt{1+4 a}) / 2$. Оно попадает в множество $\mathbf{K}$ только при

$$
a=6+10 l+4 l^{2}, \quad l=0,1,2, \ldots
$$

Если $a$ отлично от значений (4.15), то поскольку здесь опять вьполнены равенства (4.4) и $Q_{3}^{\prime}$ отсутствует, множество $\mathbf{S}_{+}^{\prime}$ порождается векторами $Q_{1}^{\prime}, Q_{6}^{\prime},\left(k_{1},-1\right)$ и состоит из векторов $Q=j Q_{1}^{\prime}+l Q_{6}^{\prime}+m\left(k_{1},-1\right)$, где целые $j, l, m \geqslant 0$. Согласно $\left(4.13^{\prime}\right)$ для этих точек $Q=\left(q_{1}, q_{2}\right)$ имеем $q_{1}=-2 j+4 l+m k_{1}, q_{2}=j-l-m$. На прямой $q_{2}=-1$ имеем $j=l+m-1$, где $m+l>0$. Поэтому $q_{1}=-2(l+m-1)+4 l+$ $m k_{1}=2+2 l+m\left(k_{1}-2\right)$. Этот же результат следует из предложения 3.3. Итак,

$$
\mathbf{K}\left(k_{1}\right)=\left\{k=2+2 l+m\left(k_{1}-2\right) ; \text { целые } l, m \geqslant 0, l+m>0\right\},
$$

и разложение $(1.12),(3.1)$ для полусемейства $\mathscr{G}_{1}^{(1)} 1+$ имеет вид

$$
y=c x^{2}+\sum_{k \in \mathbf{K}\left(k_{1}\right)} c_{k} x^{k}
$$

где $c=\sqrt{a^{-1}}, c_{k_{1}}-$ произвольньй постоянньй коэффициент и все остальные коэффициенты $c_{k}$ постоянны и однозначно определены.

Если вьполнено равенство (4.15), то $\mathbf{K}\left(k_{1}\right)=\mathbf{K}$, но разложение (1.12), (3.5) для полусемейства $\mathscr{G}_{1}^{(1)} 1+$ содержит логарифмы для $\beta_{k}$ с $k \geqslant 4+2 l=k_{1}$, где $l$ из равенства (4.15). При этом коэффициент $\beta_{k_{1}}$ содержит произвольную постоянную. Например, при $l=0$ имеем $a=6, k_{1}=4$. Поэтому разложение $(1.12),(3.5)$ имеет вид

$$
\begin{gathered}
y=c x^{2}+\beta x^{4}+\cdots, \\
y^{\prime}=2 c x+4 \beta x^{3}+\beta^{\prime} x^{4}+\cdots, \quad y^{\prime \prime}=2 c+12 \beta x^{2}+8 \beta^{\prime} x^{3}+\beta^{\prime \prime} x^{4}+\cdots .
\end{gathered}
$$

Подставляя эти выражения в (2.1) и приравнивая к нулю коэффициент при $x^{6}$, получаем уравнение для $\beta$

$$
6 \beta^{\prime} x+\beta^{\prime \prime} x^{2}=c / 2 .
$$

Оно имеет решения

$$
\beta=(c / 12) \ln x+\tilde{c},
$$

где $\tilde{c}$ - произвольная постоянная. Следовательно,

$$
y=\sqrt{a^{-1}} x^{2}+\left[\left(\sqrt{a^{-1}} / 12\right) \ln x+\tilde{c}\right] x^{4}+\cdots .
$$


Теперь вьгислим $\beta$, применяя замечание 3.1. Подставим $y=c x^{2}$ в уравнение $(2.1)$ и выделим коэффициент при $x^{6}$, ибо здесь $v=2$ и $k=4$, т.е. $k+v=6$. Получаем $\theta_{4}=c^{2}$. Согласно $\left(2.16^{\prime}\right)$ при $a=6$

$$
\nu(k)=-2 c\left[k^{2}-3 k-4\right],
$$

т.е. $\nu^{\prime}(k)=-2 c(2 k-3), \nu^{\prime \prime}(k)=-4 c$. При $k=4$ формула (3.7) принимает вид

$$
\nu(4) \beta_{4}+\nu^{\prime}(4) \dot{\beta}_{4}+\frac{1}{2} \nu^{\prime \prime}(4) \ddot{\beta}_{4}+c^{2}=0,
$$

т.е. $-10 c \dot{\beta}_{4}-2 \ddot{\beta}_{4}=c^{2}$, что согласно $\left(4.9^{\prime}\right)$ аналогично $\left(4.17^{\prime}\right)$. Здесь $\lambda(4)=0, \mu(4)=1$ и $\lambda+\mu=1$. По лемме 3.2 многочлен $\beta_{4}(\xi)$ имеет первую степень, т.е. $\ddot{\beta}_{4}=0$.

Итак, при $a>0$ полусемейство $\mathscr{G}_{1}^{(1)} 1+$, если не выполнено ни одно из равенств (4.15), состоит из разложений (4.17), (4.16), где постоянньй коэффициент $c_{k_{1}}$ произволен, а если выполнено одно из равенств (4.15), то оно состоит из разложений (1.12), $(3.5)$, где $k=4+2 l, l=0,1,2, \ldots$ Полусемейство $\mathscr{G}_{1}^{(1)} 2+$ устроено аналогично, оно получается из полусемейства $\mathscr{G}_{1}^{(1)} 1+$ заменой $\sqrt{a^{-1}}$ на $-\sqrt{a^{-1}}$.

4.6. Семейства $\mathscr{F}_{3}^{(1)} 1$ и $\mathscr{F}_{3}^{(1)} 2$ из (2.19). Для них $r=1$ и $\omega=1$, т.е. разложение по убьваюшим степеням $x$. Носитель $\mathbf{S}(\tilde{f})$ состоит из точек

$$
Q_{1}, \quad Q_{2}, \quad Q_{4}=(1,1), \quad Q_{5}=(3,1), \quad Q_{7}=(2,0)
$$

Члену $\mathscr{L}(x) z$ соответствует вершина $Q_{5}$. Положим $Q_{i}^{\prime}=Q_{i}-Q_{5}$, тогда

$$
Q_{1}^{\prime}=(-3,1), \quad Q_{2}^{\prime}=(-1,1), \quad Q_{4}^{\prime}=(-2,0), \quad Q_{5}^{\prime}=(0,0), \quad Q_{7}^{\prime}=(-1,-1) .
$$

Все эти точки лежат в решетке с базисом $B_{1}=(1,1), B_{2}=(2,0)$. Поэтому множество $\mathbf{K}=\{k=-1-2 l, l=0,1,2, \ldots\}$. Следовательно, разложения (1.12), (3.5) для семейств $\mathscr{G}_{3}^{(1)} 1$ и $\mathscr{G}_{3}^{(1)} 2$ имеют вид

$$
y=c x+x^{-1} \sum_{k=0}^{\infty} c_{-2 k} x^{-2 k}
$$

где $c= \pm 1$ и все $c_{-2 k}$ однозначно определены для фиксированного $c$.

4.7. Сводка результатов. В этом параграфе найдены следующие разложения решений уравнения (2.1).

I. Двупараметрическое (по $c$ и $a$ ) семейство $\mathscr{G}_{1}^{(0)} 1$ степенных разложений $(3.1)$ с показателями (4.6).

II. Трехпараметрическое (по $c, c_{1}$ и $а$ ) семейство $\mathscr{G}_{1}^{(0)} 2$ степенно-логарифмических разложений (4.10). Если не выполнено равенство (4.7), то это семейство степенных разложений (3.1) с множеством показателей (4.8).

III. Однопараметрическое (по $c$ ) семейство $\mathscr{G}_{1}^{(0)} 3$ степенных разложений (4.11).

IV. Двупараметрическое (по $c$ и $a$ ) семейство $\mathscr{G}_{1}^{(0)} 4$ степенных разложений $(1.20)$, (4.13) с комплексными показателями из множества (4.12) и комплексно-сопряженное ему семейство $\mathscr{G}_{1}^{(0)} 5$. 
V. Два однопараметрических (по $a<0)$ полусемейства $\mathscr{G}_{1}^{(1)} 1-$ и $\mathscr{G}_{1}^{(1)} 2-$ степенных разложений (4.14).

VI. Два двупараметрических (по $a>0$ и $c_{k_{1}}$ ) семейства $\mathscr{G}_{1}^{(1)} 1+$ и $\mathscr{G}_{1}^{(1)} 2+$ степенно-логарифмичческих разложений (1.12), (3.5). Если не вьполнены равенства (4.15), то они состоят из степенных разложений (4.17) с множеством показателей (4.16).

VII. Два однопараметрических (по $a)$ семейства $\mathscr{G}_{3}^{(1)} 1$ и $\mathscr{G}_{3}^{(1)} 2$ степенных разложений (4.18).

\section{§5. Нестепенные асимптотики решений}

5.1. Постановка задачи. Пусть $x \rightarrow 0$ или $x \rightarrow \infty$. Две функции $\varphi(x)$ и $\psi(x)$ называются слабо (асимптотически) эквивалентными, если

$$
\psi(x) / \varphi(x) \rightarrow 1 .
$$

При этом функция $\varphi(x)$ является слабой асимптотикой функции $\psi(x)$ и наоборот. Обозначим $k$-кратные экспоненту и логарифм через $\exp ^{(k)} x \ln ^{(k)} x$ соответственно, т.e.

$$
\exp ^{(k)} x \stackrel{\text { def }}{=} \exp (\exp (\ldots(\exp x) \ldots)) \text { и } \ln ^{(k)} x \stackrel{\text { def }}{=} \ln (\ln (\ldots(\ln x) \ldots)) .
$$

ЗАДАчА 3. Для решений уравнения (1.4), где $f(x, y)$ - дифференциальная сумма, найти все (слабые) асимптотики вида

$$
y=c x^{r}(\exp x)^{s_{1}} \cdots\left(\exp ^{(k)} x\right)^{s_{k}}(\ln x)^{t_{1}} \cdots\left(\ln ^{(l)} x\right)^{t_{l}},
$$

где $c=$ const $\in \mathbb{C}, c \neq 0 ; r, s_{i}, t_{i}=$ const $\in \mathbb{R}$ или $\mathbb{C}$.

В $\S 1$ изложен метод вычисления всех степенных асимптотик, т.е. асимптотик вида (1.7). Поэтому здесь сосредоточимся на вычислении всех нестепенных асимптотик решений, т.е. не имеющих вида (1.7).

Порядком функиии $\varphi(x)$ назьвается число

$$
r=\lim (\ln |\varphi(x)| / \ln |x|) \in[-\infty,+\infty],
$$

если предел сушествут. Очевидно, что слабо эквивалентные функции имеют одинаковый порядок.

Две функции $\varphi(x)$ и $\psi(x)$ сильно (асимптотически) әквивалентны, если

$$
\varphi(x)=\psi(x)\left[1+o\left(|x|^{\omega \varepsilon}|\psi(x)|^{\omega_{2} \varepsilon}\right)\right]
$$

для некоторого $\varepsilon<0$, где

$$
\omega_{2}= \begin{cases}-1, & \text { если } \psi(x) \rightarrow 0, \\ 0, & \text { если } \psi(x) \rightarrow \text { const } \neq 0, \\ 1, & \text { если } \psi(x) \rightarrow \infty\end{cases}
$$

При этом функция $\varphi(x)$ является сильной асимптотикой функции $\psi(x)$ и наоборот. 
Пусть $y=\varphi(x)$ - решение уравнения (1.4) и функция $\varphi(x)$ имеет порядок $r$. Тогда нормальным конусом и этого решения является луч

$$
\mathbf{u}= \begin{cases}\lambda(0,-1), & \text { если } r=-\infty ; \\ \lambda \omega(1, r), & \text { если } r \in \mathbb{R} ; \\ \lambda(0,1), & \text { если } r=+\infty, \lambda>0 .\end{cases}
$$

Понятие конус задачи применимо и к решениям с так определенньм нормальным конусом $\mathbf{u}$.

Теорема 5.1 [2; гл. VI, теорема 1.1]. Если решение $y=\varphi(x)$ уравнения (1.4) имеет нормальный конус $\mathbf{u}$, то укороченное уравнение $(1.9)$, для которого $\mathbf{u} \subset$ $\mathrm{U}_{j}^{(d)}$, имеет сильно асимптотически әквивалентное решение $y=\psi(x)$.

Таким образом, задача 3 сводится к нахождению всех укороченных уравнений и их нормальных конусов и к конечному числу следующих задач.

ЗАДАчА 4. Для укороченного уравнения (1.9) найти все (слабые) асимптотики его решений $y=\psi(x)$ с $(1, r) \in \mathbf{N}_{j}^{(d)}$. Из этих решений надо оставить только те, у которых $\omega(1, r) \in \mathbf{U}_{j}^{(d)}$.

Согласно $\S 1$ уравнению (1.4) соответствует многоугольник $\Gamma(f)$, а укороченному уравнению (1.9) - его ребро или вершина $\Gamma_{j}^{(d)}$. Ниже рассмотрим по отдельности четыре способа редукции задачи 4: три для ребра (в зависимости от его наклона) и один для вершины.

ЗАмечАниЕ 5.1. Алгебраическое укороченное уравнение (1.9) не имеет подходяших нестепенных решений, т.е. не дает нестепенных асимптотик решений уравнения (1.4).

5.2. Случай вертикального ребра $\Gamma_{j}^{(1)}$. Если ребро $\Gamma_{j}^{(1)}$ вертикально, то его нормальный конус

$$
\mathbf{U}_{j}^{(1)}=\lambda \omega(1,0), \quad \lambda>0,
$$

и у всех точек $Q=\left(q_{1}, q_{2}\right) \in \Gamma_{j}^{(1)}$ координата $q_{1}$ одинакова. Положим

$$
g(x, y)=x^{-q_{1}} \hat{f}_{j}^{(d)}(x, y)
$$

тогда носитель $\mathbf{S}(g)$ лежит на координатной оси $q_{1}=0$. Согласно (5.2) все степенные решения $(1.7)$ с $(1, r) \in \mathbf{N}_{j}^{(1)}$ являются постоянными $y=y^{0}=$ const, где $y^{0}-$ корень определяющего уравнения

$$
\tilde{g}(y) \stackrel{\text { def }}{=} g(0, y)=0
$$

Корень $y^{0}$ уравнения (5.4) называется кратнылм, если в нем производная $d \tilde{g}(y) / d y$ равна нулю.

Для отыскания нестепенных решений уравнения (1.9) сделаем логарифмическое преобразование

$$
\xi \stackrel{\text { def }}{=} \ln x .
$$


Согласно теореме 2.4 из [2; гл. VI] при этом дифференциальная сумма $g(x, y)$ перейдет в диффференщиальную сумму $h(\xi, y) \stackrel{\text { def }}{=} g(x, y)$ и уравнение $(1.9)$ примет вид

$$
h(\xi, y)=0 .
$$

Из (5.5) видно, что $\xi \rightarrow \infty$ при $x \rightarrow 0$ и при $x \rightarrow \infty$, т.е. для уравнения (5.6) получаем задачу 3 с конусом задачи

$$
p_{1} \geqslant 0 \text {. }
$$

ТЕОРема 5.2. Конечные предельные значения $y^{0} \neq 0$ непостоянных решений уравнения (5.6) являются кратными решениями уравнения (5.4).

Пусть ребро $\Gamma_{j}^{(1)}$ соединяет вершины $\Gamma_{j-1}^{(0)}=\left(q_{1}^{\prime}, q_{2}^{\prime}\right)$ и $\Gamma_{j}^{(0)}=\left(q_{1}^{\prime}, q_{2}^{\prime \prime}\right)$, где целые $q_{2}^{\prime}<q_{2}^{\prime \prime}$. Будем говорить, что уравнение (5.4) имеет бесконечныи корень, если степень многочлена в (5.4) меньше $q_{2}^{\prime \prime} ;$ и имеет нулевой корень, если у многочлена в (5.4) наименьшая степень по $y$ больше $q_{2}^{\prime}$.

ТЕОРема 5.3. Если уравнение (5.4) не имеет бесконечного (нулевого) корня, то уравнение (5.6) не имеет решений, стремящихся к бесконечности (нулю).

Следовательно, если уравнение (5.4) не имеет кратных корней, а также бесконечного и нулевого корней, то уравнение (1.9) не имеет подходяших нестепенных решений, т.е. преобразование (5.5) и дальнейшие исследования делать не нужно. В противном случае надо сделать преобразование (5.5) и рассмотреть уравнение (5.6).

Заметим, что укорочение уравнения (5.6) относительно вектора $(1,0)$ является уравнением $(5.4)$, т.е. $\tilde{g}(y) \equiv \hat{h}(\xi, y)$. Для нахождения решений уравнения (5.6) с бесконечными и нулевьми предельными значениями $y$ надо для уравнения (5.6) выделить укороченные уравнения, соответствующие конусу задачи (5.7). Для нахождения решений уравнения (5.6) с конечными предельными значениями надо найти все кратные корни уравнения (5.4). Пусть $y^{0}$ - такой кратный корень. Тогда параллельным сдвигом $y=y^{0}+z$ надо поместить его в начало координат. Уравнение (5.6) примет вид

$$
\tilde{h}(\xi, z) \stackrel{\text { def }}{=} h\left(\xi, y^{0}+z\right)=0 .
$$

Теперь для уравнения (5.8) надо выделять его укороченные уравнения с конусом задачи $p_{1} \geqslant 0, p_{2} \leqslant 0$. Это опять задача 3 .

Итак, для вертикального ребра здесь был описан один шаг, позволяюший найти все степенные решения уравнения (1.9) и для нахождения нестепенных асимптотик его решений сводящий задачу 4 к конечному числу задач 3.

5.3. Случай наклонного ребра $\Gamma_{j}^{(1)}$. Пусть вектор $(1, r)$ с $r \neq 0$ является нормальным к ребру $\Gamma_{j}^{(1)}$.

Tеорема 5.4 [2; гл. VI, теорема 2.2]. Степенное преобразование

$$
y=z x^{r}
$$

приводит уравнение (1.9) $к$ виду

$$
\tilde{f}(x, z) \stackrel{\text { def }}{=} \hat{f}_{j}^{(1)}\left(x, z x^{r}\right)=0,
$$


әде $\tilde{f}(x, z)$ - дифференциальная сумма и ее носитель $\mathbf{S}(\tilde{f})$ расположен на вертикали $q_{1}=$ const.

Таким образом, степенным преобразованием (5.9) случай наклонного ребра сводится к случаю вертикального ребра. Если в укороченное уравнение (1.9) подставить $y=c x^{r}$, то для коэффициента $c$ получается уравнение

$$
x^{s} \tilde{\tilde{f}}(c) \stackrel{\text { def }}{=} \hat{f}_{j}^{(1)}\left(x, c x^{r}\right)=0,
$$

где $\tilde{\tilde{f}}(c)$ - многочлен от коэффициента $c$. Здесь он играет ту же роль, что и многочлен $\tilde{g}(y)$ из уравнения (5.4). В частности, если этот многочлен не имеет бесконечного, нулевого и кратных корней, то уравнение (1.9) не имеет подходящих нестепенных решений, т.е. преобразование (5.9) и дальнейшее исследование делать не надо.

СлЕДСТВИЕ 5.1. Если ребро $\Gamma_{j}^{(1)}$ не горизонтально, соответствующее ему определяющее уравнение $\tilde{\tilde{f}}(c)=0$ не имеет кратных корней $c \neq 0$ и иисло $r$ не является корнем характеристических уравнений для обеих вершин ребра $\Gamma_{j}^{(1)}$, əде $N_{j}=(1, r)$ - нормаль к ребру $\Gamma_{j}^{(1)}$, то ребру $\Gamma_{j}^{(1)}$ не соответствуют нестепеннье асимптотики.

5.4. Случай горизонтального ребра $\Gamma_{j}^{(1)}$. В этом случае у всех точек $Q=$ $\left(q_{1}, q_{2}\right)$ ребра $\Gamma_{j}^{(1)}$ координата $q_{2}$ одинакова. Положим

$$
g(x, y) \stackrel{\text { def }}{=} y^{-q_{2}} \hat{f}_{j}^{(1)}(x, y)
$$

и сделаем логарифмическое преобразование

$$
\eta=d \ln y / d x .
$$

Согласно теореме 2.4 из [2; гл. VI] при этом преобразовании дифференциальная сумма $g(x, y)$ перейдет в дифференциальную сумму $h(x, \eta) \stackrel{\text { def }}{=} g(x, y)$ и уравнение (1.9) примет вид

$$
h(x, \eta)=0 .
$$

Теперь для уравнения (5.13) получаем задачу 3 с конусом задачи

$$
p_{1}+p_{2} \geqslant 0 .
$$

Сумму порядков всех производных, входяших в дифференщиальный моном $a(x, y)$, назовем суммарным порядком дифференцирования монома $a(x, y)$ и обозначим $\Delta(a)$. Для дифференщиальной суммы (1.3) суммарный порядок дифференцирования

$$
\Delta(f)=\max _{i} \Delta\left(a_{i}\right)
$$

Пусть $\Omega_{j}$ - степень характеристического многочлена $\chi_{j}(r)$ вершины $\Gamma_{j}^{(0)}$. Пусть ребро $\Gamma_{j}^{(1)}$ соединяет вершины $\Gamma_{j-1}^{(0)}=\left(q_{1}^{\prime}, q_{2}^{\prime}\right)$ и $\Gamma_{j}^{(0)}=\left(q_{1}^{\prime \prime}, q_{2}^{\prime}\right)$ с $q_{1}^{\prime}<q_{1}^{\prime \prime}$. 
TеОрема 5.5. Если $\Omega_{j-1}=\Delta\left(\hat{f}_{j-1}^{(0)}\right)=\Delta\left(\hat{f}_{j}^{(1)}\right)\left(u л u \Omega_{j}=\Delta\left(\hat{f}_{j}^{(0)}\right)=\Delta\left(\hat{f}_{j}^{(1)}\right)\right)$, то уравнение (5.13) не имеет подходящих решений при $x \rightarrow 0($ или $x \rightarrow \infty)$.

Следовательно, если $\Omega_{j-1}=\Delta\left(\hat{f}_{j-1}^{(0)}\right)=\Delta\left(\hat{f}_{j}^{(1)}\right)=\Delta\left(\hat{f}_{j}^{(0)}\right)=\Omega_{j}$, то преобразование $(5.12)$ и дальнейшее исследование делать не надо.

5.5. Случай вершины $\Gamma_{j}^{(0)}$. Пусть $\Gamma_{j}^{(0)}=\{Q\}$. Положим

$$
g(x, y) \stackrel{\text { def }}{=} X^{-Q} \hat{f}_{j}^{(0)}(x, y)
$$

тогда $\mathbf{S}(g)=\{0\}$. Степенные решения $y=c x^{r}$ уравнения $g(x, y)=0$ имеют произвольную постоянную $c$ и показатель $r$, удовлетворяющий характеристическому уравнению

$$
\chi(r) \stackrel{\text { def }}{=} g\left(x, x^{r}\right)=0 .
$$

Для нахождения нестепенных решений сделаем логарифмическое преобразование

$$
\xi=\ln x, \quad \eta=d \ln y / d \ln x .
$$

Согласно теореме 2.4 из [2; гл. VI] при этом дифференщиальная сумма $g(x, y)$ перейдет в дифференщиальную сумму $h(\xi, \eta) \stackrel{\text { def }}{=} g(x, y)$ и уравнение $(1.9)$ примет вид

$$
h(\xi, \eta)=0
$$

Из (5.16) видно, что $\xi \rightarrow \infty$, т.е. $p_{1} \geqslant 0$. Таким образом, для уравнения (5.17) получаем задачу 3 с конусом задачи $p_{1} \geqslant 0, p_{1}+p_{2} \geqslant 0$. Теперь заметим, что согласно (5.16) степенному решению (1.7) уравнения (1.9) соответствует постоянное решение

$$
\eta=r=\text { const }
$$

уравнения (5.17)

ТЕОРема 5.6. Конечные предельные значения $\eta^{0} \neq 0$ непостоянных решений уравнения (5.17) являются кратньм.ми корнями уравнения (5.15).

Пусть $\Delta(g(x, y))=k$. Обозначим через $g^{*}(x, y)$ сумму всех тех дифференциальных мономов суммы $g(x, y)$, у которых суммарньй порядок дифференцирования равен $k$, т.е. максимален в $g(x, y)$. Через $\operatorname{coef}\left(g^{*}\right)$ обозначим сумму всех числовых коэффициентов в дифференциальных мономах суммы $g^{*}(x, y)$.

ТЕОРема 5.7. Если дифференциальная сумма $g(x, y)$ из (5.14) содержит ненулевую постоянную (или для нее $\left.\operatorname{coef}\left(g^{*}\right) \neq 0\right)$, то уравнение $(5.17)$ не имеет решений, стремящихся к нулю (или к бесконечности). 
СЛЕДСТВИЕ 5.2. Пусть характеристический многочлен $\chi_{j}(r)$ для вершинь $\Gamma_{j}^{(0)}$ не имеет таких кратных корней $r$, для которых хотя бы один из векторов $\pm(1, r)$ лежст в нормальном конусе $\mathrm{U}_{j}^{(0)}$, и если один из векторов $\pm(0,1)$ лежит в $\mathrm{U}_{j}^{(0)}$, то $\Omega_{j}=\Delta\left(\hat{f}_{j}^{(0)}\right)$, т.е. многочлен $\chi_{j}(r)$ не имеет бесконечного корня. Тогда вериине $\Gamma_{j}^{(0)}$ не соответствуют нестепенные асимптотики.

Следовательно, если для $\hat{f}_{j}^{(0)}(x, y)$ характеристическое уравнение не имеет кратных корней, сумма $g(x, y)$ имеет ненулевой свободньй член и $\operatorname{coef}\left(g^{*}\right) \neq 0$, то уравнение (1.9) не имеет подходяших нестепенных решений.

Заметим, что укорочение уравнения (5.17) относительно вектора $(1,0)$ является уравнением $(5.15)$, т.е. $\chi(\eta) \equiv \hat{h}(\xi, \eta)$. Для нахождения решений уравнения $(5.17)$ с бесконечными и нулевыми предельными значениями надо для уравнения (5.17) выделять укороченные уравнения, у которых нормальньй конус пересекается с конусом задачи $p_{1} \geqslant 0, p_{1}+p_{2} \geqslant 0$ и т. д. Для нахождения решений уравнения $(5.17)$ с конечными ненулевыми предельными значениями надо найти все кратные корни уравнения (5.15). Пусть $\eta^{0}$ - такой корень. Параллельным переносом $\eta=\eta^{0}+\zeta$ надо поместить его в начало координат. Уравнение (5.17) примет вид

$$
\tilde{h}(\xi, \zeta) \stackrel{\text { def }}{=} h\left(\xi, \eta^{0}+\zeta\right)=0 .
$$

Теперь надо выделять укорочения уравнения (5.19) с конусом задачи $p_{1} \geqslant 0, p_{2} \leqslant 0$. Это опять задача 3.

ЗАмечАниЕ 5.2. Доказательства теорем 5.2-5.7 основаны на рассмотрении спешифики многоугольника $\Gamma(h)$, получаюшегося после логарифмического преобразования. При этом преобразования (5.12) и (5.16) удобнее делать в два этапа: сначала ввести логарифм $w=\ln y$ зависимой переменной $y$, а затем ввести его производную $\eta=d w / d x$ в (5.12) и $\eta=d w / d \xi$ в (5.16). Кроме того, этим способом легко доказать, что после преобразования (5.12) или (5.16) конус задачи лежит в множестве $p_{1}+p_{2} \geqslant 0$.

5.6. Последовательность редукций. Итак, в пш. 5.2-5.5 был описан один шаг, позволяюший найти все степенные асимптотики решений уравнения (1.4) и для нахождения нестепенных асимптотик сводящий задачу 3 к конечному числу задач 3 для преобразованных уравнений вида (1.4). Каждая из них имеет конечное число задач 4 для укороченных уравнений вида (1.9).

ТЕОРема 5.8. Через конечное число таких шагов получается укороченная система, имеющая только степенные подходящие решения.

Последовательность таких вычислений состоит в том, что по исходному уравнению (1.4) вычисляется многоугольник $\Gamma(f)$, его грани $\Gamma_{j}^{(d)}$ и соответствующие укороченные уравнения (1.9). Согласно теореме 5.1 точное решение укороченного уравнения (1.9) является сильной асимптотикой решений исходного уравнения (1.4). Сначала находим все семейства $\mathscr{F}_{j}^{(d)} k$ степенных решений уравнения (1.9). Вблизи каждого невырожденного семейства $\mathscr{F}_{j}^{(d)} k$ методами $\S 3$ можно получить семейство $\mathscr{G}_{j}^{(d)} k$ степенно-логарифмических разложений решений исходного уравнения (1.4). Согласно 
теоремам 5.2-5.7 укороченное уравнение (1.9) может иметь подходящие нестепенные решения только вблизи семейств $\mathscr{F}_{j}^{(d)} k$, соответствуюших кратным корням определяющего уравнения $\tilde{\tilde{f}}(c)=0$ или характеристического уравнения $\chi(r)=0$, а также вблизи их нулевого корня (что обозначим через $\left.\mathscr{F}_{j}^{(d)} 0\right)$ и вблизи их бесконечного корня (что обозначим через $\mathscr{F}_{j}^{(d)} \infty$ ). Если согласно теоремам 5.2-5.7 такие решения могут быть, то после соответствуюших степенного преобразования, логарифмического преобразования и сдвига из уравнения (1.9) для каждого случая $\mathscr{F}_{j}^{(d)} k$ получаем свою задачу 3 со своим уравнением $\tilde{h}(\xi, \eta)=0$, своим многоугольником $\widetilde{\Gamma}=\Gamma(\tilde{h})$ и своим конусом задачи. Пусть $\widetilde{\Gamma}_{j_{1}}^{\left(d_{1}\right)}-$ грань этого многоугольника. Соответствующие ей семейства степенных решений обозначим как $\mathscr{F}_{j}^{(d)} k \mathscr{F}_{j_{1}}^{\left(d_{1}\right)} k_{1}$. Каждому такому семейству соответствует семейство $\mathscr{F}_{j}^{(d)} k_{j_{1}}^{\left(d_{1}\right)} k_{1}$ разложений решений уравнения $\tilde{h}(\xi, \eta)=0$. Ему соответствуют семейство нестепенных решений уравнения (1.9) и семейство нестепенных асимптотик решений исходного уравнения (1.4), для которых будем использовать те же обозначения. Эту процедуру можно продолжать и получать семейства вида

$$
\mathscr{F}_{j}^{(d)} k \mathscr{F}_{j_{1}}^{\left(d_{1}\right)} k_{1} \ldots \mathscr{F}_{j_{l-1}}^{\left(d_{l-1}\right)} k_{l-1} \mathscr{F}_{j_{l}}^{\left(d_{l}\right)} k_{l}
$$

и вида

$$
\mathscr{F}_{j}^{(d)} k \mathscr{F}_{j_{1}}^{\left(d_{1}\right)} k_{1} \cdots \mathscr{F}_{j_{l-1}}^{\left(d_{l-1}\right)} k_{l-1} \mathscr{G}_{j_{l}}^{\left(d_{l}\right)} k_{l},
$$

где $k$ и $k_{i}$ принимают значения из множества $\{0\} \cup \mathbb{N} \cup\{\infty\}$. Если проделать обратные преобразования и вернуться к исходным координатам $x, y$, то при $l=1$ семейство (5.20) дает слабые асимптотики вида (5.1), а семейство (5.21) - сильные. Вообще семейства (5.20) дают выражения вида (5.1). При $l>1$ они являются слабыми асимптотиками для $\ln ^{(l-1)} y$. Согласно теореме 5.8 число $l$ в семействах $(5.20)$ и $(5.21)$ не превосходит некоторой константы, определяемой исходньм уравнением (1.4). При этом чем больший отрезок разложения семейства $\mathscr{G}_{j_{l}}^{\left(d_{l}\right)} k_{l}$ в $(5.21)$ удастся вьчислить, тем точнее будет найдена асимптотика решений исходного уравнения (1.4).

ЗАмечАниЕ 5.3. Степенные и логарифмические преобразования, описанные в этом параграфе, сильно упрошают уравнение. Может случиться, что преобразованное уравнение решается в явном виде [6]. В этом случае можно не вычислять разложения его решений.

\section{§. Примеры вычисления нестепенных асимптотик}

Рассмотрим уравнение (2.1) и найдем нестепенные асимптотики его решений, соответствуюшие вершинам $\Gamma_{j}^{(0)}$ и ребрам $\Gamma_{j}^{(1)}$.

6.1. Вершина $\Gamma_{1}^{(0)}$ (п. 2.2). Ей соответствует укороченное уравнение $(2.5)$ и $\Gamma_{1}^{(0)}=$ $(0,2)$. Действуем согласно п. 5.6. Положим

$$
g(x, y) \stackrel{\text { def }}{=} y^{-2} \hat{f}_{1}^{(0)}(x, y)=x^{2} y^{-2} y^{\prime 2}-2 x^{2} y^{-1} y^{\prime \prime}+a=0 .
$$

Характеристическое уравнение (2.6) имеет кратные корни $\left(r_{1,2}=1\right)$ только при $a=-1$. Для суммы $g(x, y)$ из (6.1) имеем $\Delta(g)=2, g^{*}=x^{2} y^{-2} y^{\prime 2}-2 x^{2} y^{-1} y^{\prime \prime}$, 
$\operatorname{coef}\left(g^{*}\right)=1-2=-1 \neq 0$. В сумме $g(x, y)$ из (6.1) постоянная (свободньй член) равна $a$. Согласно теоремам 5.6 и 5.7 при $a \neq-1$ и $a \neq 0$ уравнение (6.1) не имеет подходящих нестепенных решений, но при $a=-1$ и при $a=0$ могут быть такие решения.

Логарифмическое преобразование (5.16) сделаем по частям. Сначалаположим $\xi=$ $\ln x$ и производную по $\xi$ будем обозначать точкой: $d y / d \xi \stackrel{\text { def }}{=} \dot{y}$. Тогда

$$
y^{\prime}=\dot{y} x^{-1}, \quad y^{\prime \prime}=(\ddot{y}-\dot{y}) x^{-2} .
$$

Уравнение (6.1) принимает вид

$$
y^{-2} \dot{y}^{2}-2 y^{-1}(\ddot{y}-\dot{y})+a=0 .
$$

Теперь положим $\eta=(\ln y)=d \ln y / d \xi$. Тогда

$$
\dot{y}=\eta y, \quad \ddot{y}=\dot{\eta} y+\eta^{2} y
$$

Уравнение (6.3) принимает вид

$$
h(\xi, \eta) \stackrel{\text { def }}{=} \eta^{2}-2\left(\dot{\eta}+\eta^{2}-\eta\right)+a=0
$$

или

$$
h(\xi, \eta) \stackrel{\text { def }}{=}-2 \dot{\eta}+2 \eta-\eta^{2}+a=0
$$

с конусом задачи

$$
p_{1} \geqslant 0, \quad p_{1}+p_{2} \geqslant 0 .
$$

Теперь по отдельности рассмотрим два случая $a=-1$ и $a=0$.

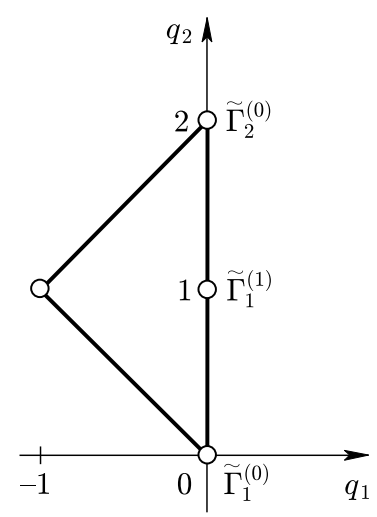

Рис. 8. 


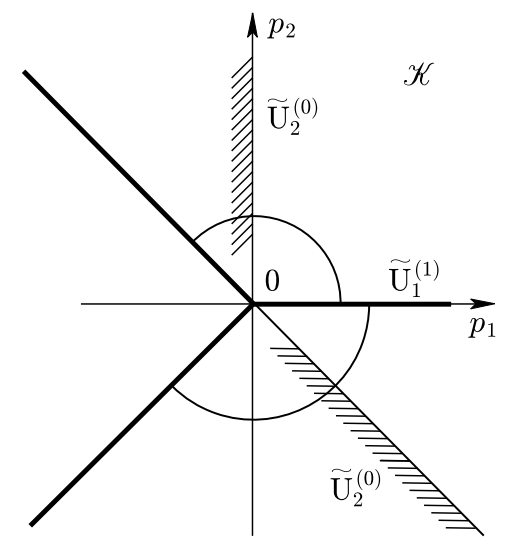

Рис. 9.

Если $a=-1$, то носитель и многоугольник уравнения (6.5) показаны на рис. 8, а нормальные конусы и конус задачи (6.6) - на рис. 9. Из него видно, что с конусом задачи (6.6) пересекаются только нормальные конусы $\mathbf{U}_{1}^{(0)}, \mathbf{U}_{2}^{(0)}$ и $\mathbf{U}_{1}^{(1)}$. Однако укороченные уравнения $\hat{h}_{1}^{(0)} \stackrel{\text { def }}{=}-1=0$ и $\hat{h}_{2}^{(0)} \stackrel{\text { def }}{=}-\eta^{2}=0$ не имеют подходяших решений. Следовательно, уравнение (6.5) не имеет подходящих решений с $\eta \rightarrow \infty$ и $\eta \rightarrow 0$. Наконец, уравнение $\hat{h}_{1}^{(1)} \stackrel{\text { def }}{=} 2 \eta-\eta^{2}-1=0$ имеет кратное решение

$$
\eta^{0}=1
$$

которое согласуется с (2.7).

Сделаем параллельньй перенос

$$
\eta=1+\zeta
$$

Тогда уравнение (6.5) при $a=-1$ примет вид

$$
\tilde{h}(\xi, \zeta) \stackrel{\text { def }}{=}-2 \dot{\zeta}-\zeta^{2}=0 .
$$

Это уравнение имеет тривиальное решение

$$
\zeta=0
$$

Носитель уравнения (6.9) состоит из двух точек $(-1,1),(0,2)$. Их вьпуклая оболочка - это отрезок с нормальным вектором $(1,-1)$. Поэтому будем искать степенное решение уравнения $(6.9)$ в виде $\zeta=\tilde{c} \xi^{-1}$. Подставляя это выражение в (6.9) и сокращая на $\xi^{-2}$, получаем алгебраическое уравнение $2 \tilde{c}-\tilde{c}^{2}=0$. Следовательно, $\tilde{c}=2$ и уравнение (6.9) имеет еше степенное решение

$$
\zeta=2 \xi^{-1}
$$


Возвращаясь по преобразованиям (6.8) и (5.16) к исходньм координатам $x, y$, получаем из решений (6.10) и (6.11) решения (2.9) и

$$
y=c x(\ln x)^{2}, \quad 0 \neq c=\text { const } \in \mathbb{C} .
$$

Эту слабую асимптотику вида $\mathscr{F}_{1}^{(0)} 3 \mathscr{F}_{1}^{(1)} 1$ можно еще уточнять, вычисляя для уравнения (6.9) решения, отличные от (6.10) и (6.11). Это можно сделать двумя способами:

a) вычисляя степенное разложение решений по убьваюшим степеням $\xi$, начинающееся с (6.11),

$$
\zeta=2 \xi^{-1}+\sum_{k=2}^{\infty} \tilde{c}_{k} \xi^{-k}
$$

получаем сильную асимптотику вида $\mathscr{F}_{1}^{(0)} 3 \mathscr{G}_{1}^{(1)} 1$; это оставляем для читателя как упражнение;

б) решая уравнение (6.9) в явном виде с помощью разделения переменных, получаем $\zeta=2(\xi+\tilde{c})^{-1}$, где $\tilde{c}-$ произвольная постоянная. Согласно (6.8) и (5.16) получаем

$$
y=c x(\ln x+\tilde{c})^{2},
$$

т.е. двупараметрическое семейство асимптотик в конечном виде, уточняющее однопараметрическое семейство нестепенных асимптотик (6.12) (ср. с замечанием 5.3).

Если $a=0$, то многоугольник уравнения (6.5) является треугольником. С конусом задачи (6.6) пересекаются только нормальные конусы укорочений $\hat{h}=2 \eta, \hat{h}=-\eta^{2}$ и $\hat{h}=2 \eta-\eta^{2}$, соответствующих двум вершинам и одному ребру. Соответствуюшие укороченные уравнения не имеют подходящих решений.

Вершинам $\Gamma_{2}^{(0)}$ и $\Gamma_{3}^{(0)}$ соответствуют алгебраические укороченные уравнения. Согласно замечанию 5.1 они не дают нестепенных асимптотик.

6.2. Ребро $\Gamma_{1}^{(1)}$ (п. 2.3). Ему соответствует укороченное уравнение (2.14). Действуем сначала согласно п. 5.3. Определяюшим уравнением (5.10) здесь является уравнение (2.15). Оно не имеет нулевого корня и конечных кратных корней. Но имеет бесконечньй (двукратньй) корень при $a=0$. Рассмотрим этот случай подробнее. Нормальным к ребру $\Gamma_{1}^{(1)}$ является вектор $(1,2)$. Поэтому степенное преобразование (5.9) есть

$$
y=x^{2} z .
$$

Поскольку

$$
y^{\prime}=2 x z+x^{2} z^{\prime}, \quad y^{\prime \prime}=2 z+4 x z^{\prime}+x^{2} z^{\prime \prime},
$$

то уравнение (2.14) при $a=0$ принимает вид

$$
\tilde{f}(x, z)=x^{4}\left[\left(2 z+x z^{\prime}\right)^{2}-2 z\left(2 z+4 x z^{\prime}+x^{2} z^{\prime \prime}\right)-1\right]=0 .
$$

После сокращения на $x^{4}$ и приведения подобных членов получаем уравнение

$$
g(x, z) \stackrel{\text { def }}{=} x^{2} z^{\prime 2}-2 x^{2} z z^{\prime \prime}-4 x z z^{\prime}-1=0 .
$$




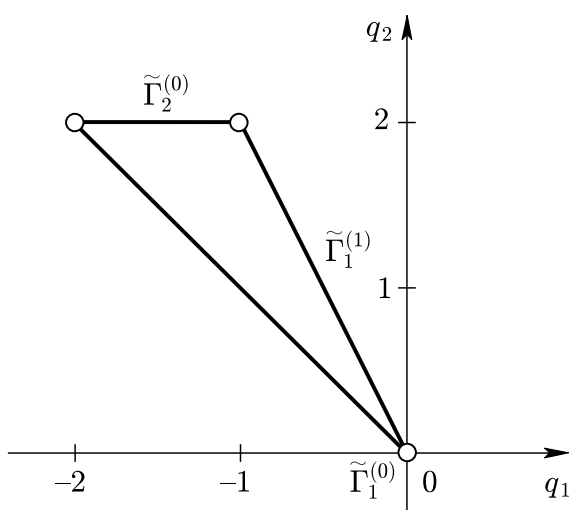

Рис. 10.

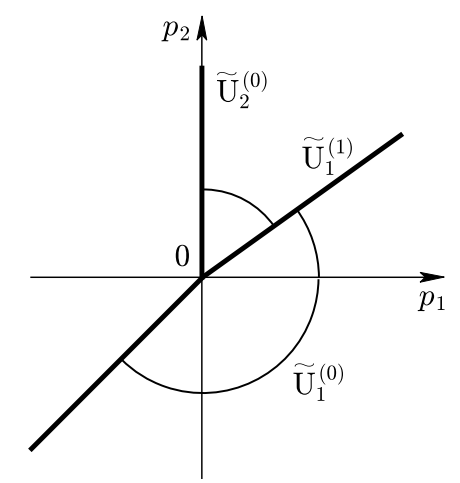

Рис. 11.

Теперь действуем согласно п. 5.2. Делаем логарифмическое преобразование (5.5). Согласно (6.2) с заменой $y$ на $z$ уравнение (6.15) принимает вид

$$
h(\xi, z) \stackrel{\text { def }}{=} \dot{z}^{2}-2 z \ddot{z}-2 z \dot{z}-1=0 .
$$

Здесь конус задачи есть $p_{1} \geqslant 0$, ибо $\xi \rightarrow \infty$, и разложение решения $z(\xi)$ должно быть по убьвающим степеням $\xi$. Носитель и многоугольник $\widetilde{\Gamma}$ уравнения (6.16) показаны на рис. 10. Нормальные конусы показаны на рис. 11. С конусом задачи $p_{1} \geqslant 0$ пересекаются три нормальных конуса $\widetilde{\mathbf{U}}_{1}^{(0)}, \widetilde{\mathbf{U}}_{2}^{(0)}$ и $\widetilde{\mathbf{U}}_{1}^{(1)}$. Но укороченное уравнение $\hat{h}_{1}^{(0)} \stackrel{\text { def }}{=}-1=0$, соответствуюшее вершине $\widetilde{\Gamma}_{1}^{(0)}=0$, не имеет решений. Укороченное уравнение $\hat{h}_{2}^{(0)} \stackrel{\text { def }}{=}-2 z \dot{z}=0$, соответствуюшее вершине $\widetilde{\Gamma}_{2}^{(0)}=(-1,2)$, имеет решение $z=$ const с нормальным вектором $(1,0)$, который не пересекается с нормальным конусом $\widetilde{\mathbf{U}}_{2}^{(0)}$. Следовательно, это решение не годится. Ребру $\widetilde{\Gamma}_{1}^{(1)}$ соответствует укороченное уравнение

$$
\hat{h}_{1}^{(1)} \stackrel{\text { def }}{=}-2 z \dot{z}-1=0 .
$$

Поскольку нормальный вектор к ребру $\widetilde{\Gamma}_{1}^{(1)}$ есть $(1,1 / 2)$, то для уравнения $(6.17)$ ищем степенное решение вида

$$
z=\tilde{c} \xi^{1 / 2} \text {. }
$$


Подставляя его в уравнение (6.17), получаем определяющее уравнение $-\tilde{c}^{2}-1=0$. Оно имеет два корня

$$
\tilde{c}_{1,2}= \pm i
$$

Согласно (6.14), (5.5), (6.18), (6.19) получаем две слабые нестепенные асимптотики

$$
y= \pm i x^{2}(\ln x)^{1 / 2}
$$

вида $\mathscr{F}_{1}^{(1)} \infty \mathscr{F}_{1}^{(1)} k, k=1,2$. Вычислим собственные значения решения (6.18) уравнения (6.17). Производная Фреше

$$
\frac{\delta \hat{h}_{1}^{(1)}}{\delta z}=-2 z \frac{d}{d \xi}-2 \dot{z}
$$

На решении (6.18) она дает оператор

$$
\mathscr{L}(\xi)=-\frac{\tilde{c}}{\xi^{1 / 2}}\left(2 \frac{d}{d \xi}+1\right) .
$$

Следовательно, $\nu(k)=-\tilde{c}(2 k+1)$, и решение (6.18), (6.19) имеет собственное значение $k_{1}=-1 / 2$. Поскольку $k_{1}<1 / 2$, то $k_{1}$ является критическим значением. Если в уравнении (6.16) сделать замену

$$
z=\tilde{c} \xi^{1 / 2}+u
$$

то получим уравнение $\tilde{h}(\xi, u) \stackrel{\text { def }}{=} h(\xi, z)=0$ с носителем, состояшим из пяти точек:

$$
\tilde{Q}_{1}=(-2,2), \quad \tilde{Q}_{2}=(-1,2), \quad \tilde{Q}_{3}=(-3 / 2,1), \quad \tilde{Q}_{4}=(-1 / 2,1), \quad \tilde{Q}_{5}=(-1,0)
$$

(рис. 12). Согласно (6.21), члену $\mathscr{L}(\xi) u$ в $\tilde{h}$ соответствует точка $\tilde{Q}_{4}$. Положим $\tilde{Q}_{i}^{\prime}=$ $\tilde{Q}_{i}-\tilde{Q}_{4}, i=1, \ldots, 5$. Все эти точки $\tilde{Q}_{i}^{\prime}$ лежат в решетке $\mathbf{Z}$ с базисом $B_{1}=(1,0)$ и $B_{2}=(1 / 2,1)$. Точка $\left(k_{1},-1\right)$ также лежит в этой решетке. С множеством $q_{1}<1 / 2$ на прямой $q_{2}=-1$ решетка $\mathbf{Z}$ пересекается по точкам $q_{1}=-1 / 2-l$, где целое $l \geqslant 0$. Следовательно, разложение решения уравнения (6.16) имеет вид

$$
z=\tilde{c} \xi^{1 / 2}+\beta \xi^{-1 / 2}+\xi^{-1 / 2} \sum_{l=1}^{\infty} \beta_{l} \xi^{-l},
$$

где $\beta$ и $\beta_{l}$ суть многочлены от $\ln \xi$. Вычислим функцию $\beta(\ln \xi)$. Дифференцируя (6.23) по $\xi$, получаем

$$
\begin{aligned}
& \dot{z}=\frac{1}{2} \tilde{c} \xi^{-1 / 2}+\dot{\beta} \xi^{-1 / 2}-\frac{1}{2} \beta \xi^{-3 / 2}+\cdots, \\
& \ddot{z}=-\frac{1}{4} \tilde{c} \xi^{-3 / 2}+\ddot{\beta} \xi^{-1 / 2}-\dot{\beta} \xi^{-3 / 2}+\frac{3}{4} \beta \xi^{-5 / 2}+\cdots .
\end{aligned}
$$


Подставим (6.23) и эти разложения в уравнение (6.16) и вьпишем члены степеней 0 и -1 по $\xi$. Получаем

$$
\frac{1}{4} \tilde{c}^{2} \xi^{-1}-\tilde{c}^{2}+2 \tilde{c}\left(\dot{\beta}-\frac{1}{2} \beta \xi^{-1}\right)+\tilde{c} \beta \xi^{-1}-\frac{1}{2} \tilde{c}^{2} \xi^{-1}-1=2 \tilde{c} \dot{\beta}-\frac{1}{4} \tilde{c}^{2} \xi^{-1} .
$$

Приравнивая коэффищиент при $\xi^{-1}$ к нулю, получаем, что $\dot{\beta}=(\tilde{c} / 8) \xi^{-1}$. Следовательно,

$$
\beta=(\tilde{c} / 8) \ln \xi+\text { const. }
$$

Согласно (6.14) и (5.5) разложению (6.23), (6.24) в исходных координатах соответствуют два однопараметрических семейства $\mathscr{F}_{1}^{(1)} \infty \tilde{\mathscr{G}}_{1}^{(1)} k, k=1,2$, асимптотик

$$
y= \pm x^{2}(\ln x)^{1 / 2}\left[i+\left(\frac{i}{8} \ln \ln x+\tilde{\tilde{c}}\right)(\ln x)^{-1}+\cdots\right]
$$

где $\tilde{\tilde{c}}$ - произвольная постоянная, которые уточняют асимптотики (6.20). Похожие вычисления асимптотик встретились в гидродинамической задаче [33; лемма 2].

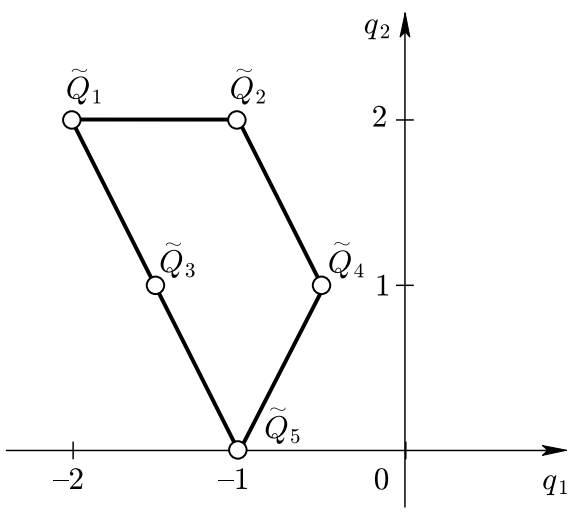

Рис. 12.

6.3. Ребро $\Gamma_{2}^{(1)}$ (п. 2.4). Ему соответствует укороченное уравнение (2.17). Действуем согласно п. 5.4. Ребро $\Gamma_{2}^{(1)}$ расположено между вершинами $\Gamma_{1}^{(0)}$ и $\Gamma_{2}^{(0)}$, которым соответствуют укороченные уравнения $(2.5)$, и $\hat{f}_{2}^{(0)} \stackrel{\text { def }}{=} x^{2} y^{2}=0$. Следовательно, порядки дифференцирования суть

$$
\Delta\left(\hat{f}_{1}^{(0)}\right)=\Delta\left(\hat{f}_{2}^{(1)}\right)=2, \quad \Delta\left(\hat{f}_{2}^{(0)}\right)=0 .
$$

По теореме 5.5 укороченное уравнение (2.17) не имеет подходящих решений при $x \rightarrow 0$, но может иметь их при $x \rightarrow \infty$. Найдем их. У всех точек $Q=\left(q_{1}, q_{2}\right)$ ребра $\Gamma_{2}^{(1)}$ координата $q_{2}=2$. Согласно (5.11) положим

$$
g(x, y) \stackrel{\text { def }}{=} y^{-2} \hat{f}_{2}^{(1)}(x, y) .
$$


Сделаем преобразование (5.12). Тогда согласно (6.4) уравнение (2.17) перейдет в уравнение вида (5.13):

$$
h(x, \eta) \stackrel{\text { def }}{=}-2 x^{2} \eta^{\prime}-x^{2} \eta^{2}+a+x^{2}=0
$$

с конусом задачи $p_{1}+p_{2} \geqslant 0$. Носитель $\mathbf{S}(h)$ и многоугольник $\Gamma(h)$ показаны на рис. 13 , а нормальные конусы - на рис. 14 . Каждой вершине соответствует укорочение $\hat{h}$, состояшее из одного обычного монома. Поэтому согласно замечанию 1.1 вершины не интересны. Что касается ребер, то согласно рис. 14 с конусом задачи $p_{1}+p_{2} \geqslant 0$ пересекаются только нормальные конусы $\widetilde{\mathbf{U}}_{1}^{(1)}$ и $\widetilde{\mathbf{U}}_{2}^{(1)}$ ребер $\widetilde{\Gamma}_{1}^{(1)}$ и $\widetilde{\Gamma}_{2}^{(1)}$. Рассмотрим их по отдельности.

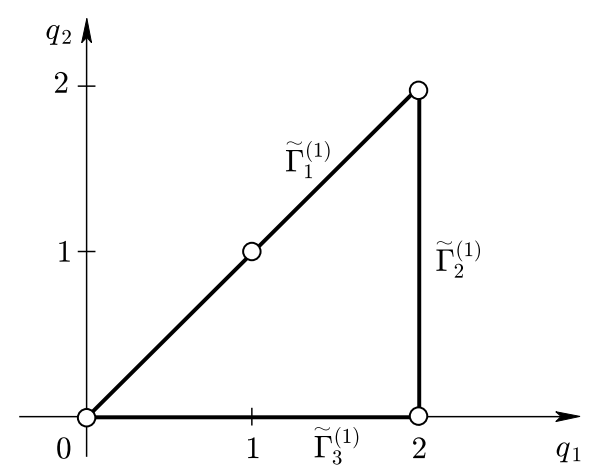

Рис. 13.

Ребру $\widetilde{\Gamma}_{1}^{(1)}$ соответствует нормальньй конус

$$
\widetilde{\mathbf{U}}_{1}^{(1)}=\{P=\lambda(-1,1), \lambda>0\}
$$

(см. рис. 14). Поскольку в нем $p_{1}<0$, то $x \rightarrow 0$, но по теореме 5.5 нет подходящих решений с $x \rightarrow 0$. Поэтому ребру $\widetilde{\Gamma}_{1}^{(1)}$ не соответствуют подходяшие решения.

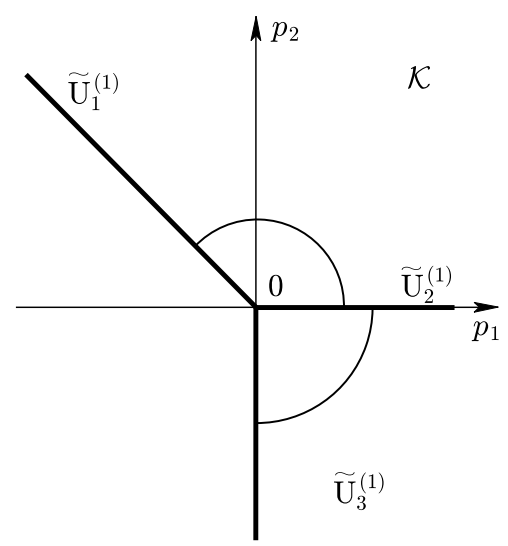

Рис. 14. 
Ребру $\widetilde{\Gamma}_{2}^{(1)}$ соответствует укороченное уравнение

$$
\hat{h}_{2}^{(1)} \stackrel{\text { def }}{=}-x^{2} \eta^{2}+x^{2}=0
$$

и нормальньй конус $p_{1}>0, p_{2}=0$ (рис. 14). Уравнение (6.28) имеет два решения

$$
\eta_{1,2}^{0}= \pm 1
$$

Согласно (5.12) им соответствуют два однопараметрических семейства $\mathscr{F}_{2}^{(1)} \infty \mathscr{F}_{2}^{(1)} k$, $k=1,2$, слабых асимптотик

$$
y=c \exp ( \pm x), \quad 0 \neq c=\text { const } \in \mathbb{C} .
$$

Поскольку укороченное уравнение (6.28) алгебраическое, то согласно замечанию 1.2 решения (6.29) не имеют критических чисел и в уравнении (6.26) им соответствуют разложения решений вида

$$
\eta=\eta^{0}+\sum_{k=2}^{\infty} c_{k} x^{-k}
$$

где все коэффициенты $c_{k}$ постоянны и однозначно определены значениями $\eta^{0}$ и $a$. Например, $c_{2}=-a /\left(2 \eta^{0}\right)$. При $a=0$ имеем в (6.31) все $c_{k}=0$. Разложениям (6.31) соответствуют сильные асимптотики

$$
y=c \exp \left[\eta^{0} x-\sum_{k=1}^{\infty}\left(c_{k+1} / k\right) x^{-k}\right], \quad \eta^{0}= \pm 1, \quad x \rightarrow \infty
$$

где $c$ произвольно, $c \neq 0$, образуюшие два двупараметрических (по $c$ и $a$ ) семейства $\mathscr{F}_{2}^{(1)} \infty \mathscr{G}_{2}^{(1)} k, k=1,2$. Они уточняют слабые асимптотики (6.30).

Ребру $\Gamma_{3}^{(1)}$ соответствует алгебраическое укороченное уравнение (2.18). Согласно замечанию 5.1 оно не дает нестепенных асимптотик.

6.4. Сводка результатов. В этом параграфе найдены следуюшие нестепенные асимптотики решений уравнения (2.1).

I. Двупараметрическое (по $c$ и $c_{1}$ ) семейство $\mathscr{F}_{1}^{(0)} 3 \mathscr{G}_{1}^{(1)} 1$ асимптотик (6.13).

II. Два однопараметрических (по $\tilde{\tilde{c}})$ семейства $\tilde{F}_{1}^{(1)} \infty \tilde{\mathscr{G}}_{1}^{(1)} 1$ и $\mathscr{F}_{1}^{(1)} \infty \tilde{\mathscr{G}}_{1}^{(1)} 2$ асимптотик (6.25).

III. Два двупараметрических (по с и а) семейства $\mathscr{F}_{2}^{(1)} \infty \mathscr{G}_{2}^{(1)} 1$ и $\mathscr{F}_{2}^{(1)} \infty \mathscr{G}_{2}^{(1)} 2$ асимптотик (6.32).

\section{$\S 7$. Асимптотически близкие решения}

7.1. Постановка задачи. Пусть задано обыкновенное дифференциальное уравнение

$$
f(x, y)=0
$$


где $f(x, y)$ - дифференциальная сумма. Каждой грани $\Gamma_{j}^{(d)}$ многоугольника $\Gamma(f)$ соответствует укороченное уравнение

$$
\hat{f}_{j}^{(d)}(x, y)=0 .
$$

Пусть $x \rightarrow 0$, тогда $\omega=-1$, или $x \rightarrow \infty$, тогда $\omega=1$. Степенные решения укороченного уравнения (7.2)

$$
y=c_{r} x^{r}, \quad c_{r}=\text { const } \in \mathbb{C}, \quad c_{r} \neq 0,
$$

с $\omega(1, r) \in \mathbf{U}_{j}^{(d)}$ являются степенными асимптотиками решений полного уравнения (7.1). Согласно $\S 1$ решению (7.3) уравнения (7.2) соответствуют: оператор

$$
\mathscr{L}(x)=\delta \hat{f}_{j}^{(d)}(x, y) / \delta y \quad \text { на } y=c_{r} x^{r}
$$

и его критические числа

$$
k_{i}, \quad k_{i} \omega<r \omega, \quad i=1, \ldots, \varkappa .
$$

Согласно $\S 3$ можно продолжить степенную асимптотику (7.3) в виде степенно-логарифмических разложений

$$
y=c_{r} x^{r}+\sum \beta_{s} x^{s} \stackrel{\text { def }}{=} \varphi(x)
$$

решений уравнения (7.1), где $\beta_{s}$ суть многочлены от $\ln x$ с комплексными коэффициентами, показатели степени $r, s \in \mathbb{R}$ и $\omega s<\omega r$.

ЗАДАчА 5. Найти все разложения вида

$$
\psi(x)=\sum \gamma_{s} x^{s}, \quad \omega s<\omega r
$$

и вида

$$
\psi(x)=\exp \sum \gamma_{s} x^{s}, \quad \omega s<\text { const },
$$

такие, что сумма $y=\varphi(x)+\psi(x)$ соответствует разложению решений уравнения (7.1). Здесь $\gamma_{s}$ суть многочлены от $\ln x$.

7.2. Степенные добавки. Здесь опишем все добавки вида (7.7) к решению (7.6).

ТеОрема 7.1. Все добавки (7.7) к решению (7.6) имеют такие показатели $s$, что $s \omega \leqslant \omega k_{i}$, где $k_{i}$ - критическое число (7.5) степенной асимптотики (7.3). При этом $\gamma_{k_{i}}(\ln x)$ - произвольный многочлен степени $l_{i}-1$, где $l_{i}$ - кратность критического числа $k_{i}$.

Наличие или отсутствие добавок (7.7) определяется по укороченному уравнению (7.2) и не требует больших вычислений.

7.3. Экспоненциальные добавки. Теперь опишем все добавки (7.8) к решению (7.6). После подстановки $y=\varphi(x)+z$ уравнение (7.1) принимает вид

$$
f(x, \varphi(x)+z) \stackrel{\text { def }}{=} \tilde{f}(x, z) \stackrel{\text { def }}{=} \mathscr{M}(x) z+g(x, z)=0
$$

где $\mathscr{M}(x)$ - линейньй дифференциальньй оператор, и у всех точек $Q=\left(q_{1}, q_{2}\right)$ носителя $\mathbf{S}(g)$ координата $q_{2} \geqslant 2$. Так что $z=0$ является решением уравнения (7.9), соответствуюшим решению (7.6) уравнения (7.1). 
ЛЕмма 7.1. В уравнении (7.9) оператор

$$
\mathscr{M}(x)=\delta f / \delta y \quad \text { на } \quad y=\varphi(x)
$$

т.е. $\mathscr{M}(x)$ это первая вариачия дифференциальной суммы $f(x, y)$, вьчисленная на решении (7.6).

Согласно $\S 3$ логарифму $\ln x$ приписьваем нулевой показатель степени. Так что произведение $\beta(\ln x) x^{r_{1}} z^{r_{2}}$, где $\beta$ - многочлен от $\ln x$, имеет показатель степени $Q=$ $\left(r_{1}, r_{2}\right)$. Теперь дифференциально-логарифмическая сумма $\tilde{f}$ имеет носитель $\mathbf{S}(\tilde{f})$ и многоугольник $\Gamma(\tilde{f})$. Многоугольник $\Gamma(\tilde{f})$ уравнения (7.9) имеет горизонтальное ребро $\Gamma_{1}^{(1)}$ с $q_{2}=1$, соответствуюшее сумме $\mathscr{M}(x) z$, т.е. $\Gamma_{1}^{(1)}=\Gamma(\mathscr{M}(x) z)$. Поэтому здесь применима техника, использованная в п. 5.4 для горизонтального ребра.

ЛЕмма 7.2. При логарифмическом преобразовании

$$
\zeta=d \ln z / d x
$$

для $l>0$ имеем

$$
z^{(l)}=z\left[\zeta^{l}+P_{l-1}\left(\zeta, \ldots, \zeta^{(l-2)}\right)\right],
$$

где штрих означает дифференцирование по $x$ и $P_{l-1}$ - многочлен степени $l-1$ от указанных переменных с постоянными коэффициентами.

Доказательство аналогично доказательству теоремы 2.2 гл. VI [2].

Для анализа решений укороченного уравнения

$$
\mathscr{M}(x) z=0
$$

соответствующего ребру $\Gamma_{1}^{(1)}$ с нормальным конусом $\mathbf{U}_{1}^{(1)}=\left\{P: p_{1}=0, p_{2}<0\right\}$, сделаем логарифмическое преобразование (7.10). Тогда уравнение (7.11) перейдет в уравнение

$$
h(x, \zeta) z \stackrel{\text { def }}{=} \mathscr{M}(x) z=0 .
$$

Разберемся в его специфике подробнее. Согласно $\S 5$ теперь для $x, \zeta$ конус задачи $\widetilde{\mathscr{K}}=$ $\left\{\tilde{P}=\left(\tilde{p}_{1}, \tilde{p}_{2}\right): \tilde{p}_{1}+\tilde{p}_{2}>0\right\}$. Многоугольник $\Gamma(h)$ обозначим $\widetilde{\Gamma}$, а его грани - как $\widetilde{\Gamma}_{j}^{(d)}$.

ЛЕмма 7.3. Всякая укороченная сумма $\hat{h}_{j}^{(d)}(x, \zeta)$, у которой нормальный конус $\tilde{\mathbf{U}}_{j}^{(d)}$ пересекается с конусом задачи $\widetilde{\mathscr{K}}$, не зависит от производньх $\zeta^{(l)} c$ $l>0$.

СлЕДСТВИЕ 7.1. Ужороченные уравнения $\hat{h}_{j}^{(0)}=0$, соответствующие вериинам $\widetilde{\Gamma}_{j}^{(0)}$, согласно замечанию 1.1 не дают подходящих решений, и их можно не рассматривать.

Укорочение $\hat{h}_{j}^{(1)}$, соответствующее ребру $\widetilde{\Gamma}_{j}^{(1)}$, является суммой нескольких членов вида

$$
b_{q, m}(\ln x) x^{q} \zeta^{m},
$$


где $b_{q, m}$ - многочлены от $\ln x$, носители которых $(q, m)=\tilde{Q}$ лежат на одном отрезке $\widetilde{\Gamma}_{j}^{(d)}$ с нормальным вектором $(\omega, p)$, где

$$
\omega+p>0
$$

Поэтому укороченное уравнение $\hat{h}_{j}^{(1)}(x, \zeta)=0$ имеет $n$ решений вида

$$
\zeta=\alpha_{i}(\ln x) x^{p \omega}, \quad i=1, \ldots, n
$$

где $n$ равно разности между наибольшим и наименшшим значениями $m$, т.е. второй координаты точек на ребре $\widetilde{\Gamma}_{j}^{(1)}$, а $\alpha_{i}$ - некоторые алгебраические функщии от $\ln x$. Решение уравнения (7.15)

$$
\ln z=\int \alpha_{i}(\ln x) x^{p \omega} d x
$$

Следовательно, решения уравнения (7.1), близкие к решению (7.6), имеют вид

$$
y=\varphi(x)+\exp \left[\int \alpha_{i}(\ln x) x^{p \omega} d x+\cdots\right]+\cdots .
$$

Если во всех членах вида (7.13), входящих в укорочение $\hat{h}_{j}^{(1)}$, коэффициенты $b_{q, m}$ постоянны, то в равенстве (7.15) функция $\alpha_{i}$ является постоянной и выражение

$$
\zeta=\alpha_{i} x^{p \omega}
$$

является подходящей степенной асимптотикой решений уравнения $h(x, \zeta)=0$. Согласно $\S 1$ степенное решение алгебраического укороченного уравнения не имеет критических чисел.

Теперь методами $§ 3$ можно получить степенно-логарифмическое разложение

$$
\zeta=\alpha_{i} x^{p \omega}+\sum \delta_{s}(\ln x) x^{s}, \quad s \omega<p
$$

решений уравнений $(7.12)$, где все многочлены $\delta_{s}$ однозначно определены. При этом значение $s=-1$ удовлетворяет неравенству $s \omega<p$ из (7.19) согласно неравенству (7.14). Поэтому разложение (7.19) дает однопараметрическое семейство решений уравнения (7.11)

$$
z=c \exp \left[\alpha_{i} x^{p \omega+1} /(p \omega+1)+\sum \gamma_{s+1}(\ln x) x^{s+1}\right]
$$

где $c$ - произвольная постоянная, $c \neq 0$ и

$$
\gamma_{s+1} x^{s+1}=\int \delta_{s} x^{s} d x
$$

Для решений уравнения (7.1) разложение (7.20) дает однопараметрическое семейство асимптотик

$$
y=\varphi(x)+c \exp \left[\alpha_{i} x^{p \omega+1} /(p \omega+1)+\sum \gamma_{s+1}(\ln x) x^{s+1}\right]+\cdots
$$


ЗАмЕЧАНИЕ 7.1. Если разложение (7.6) не содержит логарифмов, то в разложении (7.19) все $\delta_{s}$ являются постоянными, т.е. не зависят от логарифмов. Поэтому разложение (7.21) имеет вид

$$
y=\varphi(x)+c x^{\delta_{-1}} \exp \left[\alpha_{i} x^{p \omega+1} /(p \omega+1)+\sum_{s \neq-1} \delta_{s} x^{s+1} /(s+1)\right] .
$$

Для дифференциальной суммы $f(x, y)$ через $\pi(f)$ обозначим наибольший порядок производной от $y$ по $x$, имеюшийся в сумме $f(x, y)$. Аналогично, для дифференциального оператора $\mathscr{L}(x)$ через $\pi(\mathscr{L})$ обозначается наибольший порядок дифференцирования по $x$, имеющийся в операторе $\mathscr{L}(x)$.

Теорема 7.2. Решение (7.6) уравнения (7.1), соответствующее оператору $(7.4)$, имеет в точности $\pi(f)-\pi(\mathscr{L})$ однопараметрических добавок вида (7.17) или thetag7.21.

СлЕДСТВИЕ 7.2. Решение (7.6) уравнения (7.1), соответствующее укороченному уравнению (7.2), может иметь экспоненииальные добавки (7.8) только в двух случаях: (а) при $\pi\left(\hat{f}_{j}^{(d)}\right)<\pi(f)$ и (б) при $\pi\left(\hat{f}_{j}^{(d)}\right)=\pi(f)=\pi$, если уравнения (7.2) и $ә \hat{f}_{j}^{(d)} / \partial y^{(\pi)}=0$ имеют общее решение $(7.3)$, для которого один из векторов $\pm(1, r) \in \mathbf{U}_{j}^{(d)}$.

Если $\pi(\mathscr{L})=\pi(f)$ и разложение (7.6) степенное, т.е. все $\beta_{s}=$ const, то согласно теореме 3.4 разложение (7.6) сходится и согласно теореме 7.2 не имеет добавок вида (7.17) или (7.21). Если же $\pi(\mathscr{L})<\pi(f)$, то разложение $(7.6)$, как правило, расходится, но зато имеет $\pi(f)-\pi(\mathscr{L})$ однопараметрических добавок вида (7.17) или (7.21).

ГИПОТЕЗА 7.1. Если степенно-логарифмическое разложсение (7.6) решения уравнения (7.1) не имеет әкспоненциально мальх добавок в комплексной области, то оно сходится для достаточно мальи $|x|^{-\omega} \neq 0$.

ЗАмЕчАниЕ 7.2. Для нахождения всех асимптотических добавок вида (7.17) достаточно вычислить отрезок оператора $\mathscr{M}(x)$, содержаший член с максимальной производной порядка $\pi(f)$. Как правило, в силу леммы 7.1 этот член является результатом вычисления на степенной асимптотике (7.3) первой вариации того дифференциального монома $a_{i}(x, y)$ суммы $f(x, y)$, который содержит производную от $y$ наибольшего порядка $\pi(f)$. Если $Q\left(a_{i}\right)=\left(q_{1}, q_{2}\right)$, то в $\mathscr{M}(x)$ искомьй член имеет показатель $q_{1}+\left(q_{2}-1\right) r$

ЗАмечАниЕ 7.3. Здесь был рассмотрен вариант нахождения добавок (7.8) для разложения (7.6) с вешественньми показателями $r, s$. Для комплексных $r$ и $s$ все сказанное вьше сохраняется, только теперь $\omega \operatorname{Re} s<\omega \operatorname{Re} r$ и $\omega \operatorname{Re} k_{i}<\omega \operatorname{Re} r$.

ЗАмЕЧАниЕ 7.4. Если найдено разложение $\zeta(x)$ решения уравнения $h(x, \zeta)=0$ (см. (7.12)), то к нему можно найти экспоненциальную добавку так же, как к разложению (7.6) решения уравнения (7.1) находится добавка (7.8). Поскольку при каждом логарифмическом преобразовании (7.10) порядок дифференщирования в уравнении уменњшается на единицу, то через $\pi(f)$ таких шагов получим алгебраическое уравнение, а добавки будут иметь вид

$$
\exp \left\{\int \zeta(x) d x+\exp \left[\int \xi(x) d x+\exp \left(\int \eta(x) d x+\cdots\right)\right]\right\}
$$


и будут также зависеть от $\pi(f)$ произвольных постоянных. Однако последней добавкой не удается воспользоваться, ибо ее знак противоположен знаку предыдущей добавки. Поэтому в разложении (7.22) будут только $\pi(f)-1$ произвольных постоянных.

\section{§ 8. Пример вычисления экспоненциальных добавок}

Покажем, как вычислять экспоненциально малье добавки (7.8). Рассмотрим уравнение (2.1)

$$
f(X) \stackrel{\text { def }}{=} x^{2} y^{\prime 2}-2 x^{2} y y^{\prime \prime}+a y^{2}+x^{2} y^{2}-x^{4}=0,
$$

где $a$ - вещественный параметр. Очевидно, что $\pi(f)=2$. На рис. 1 показан многоугольник $\Gamma(f)$, которьй является треугольником. Из трех его вершин двум соответствуют алгебраические укороченные уравнения, которые не имеют степенных решений, а одной вершине $\Gamma_{1}^{(0)}$ соответствует укорочение $\hat{f}_{1}^{(0)}$ с $\pi\left(\hat{f}_{1}^{(0)}\right)=2$. По следствию 7.2 решения, соответствующие укороченному уравнению $\hat{f}_{1}^{(1)}=0$, не имеют экспоненциальных добавок (7.8). Из трех ребер треугольника $\Gamma(f)$ два содержат вершину $\Gamma_{1}^{(0)}$, поэтому для соответствуюших им укорочений $\hat{f}_{j}^{(1)}$ имеем $\pi\left(\hat{f}_{j}^{(1)}\right)=2$ и по следствию 7.2 для них нет добавок (7.8). Наконец, ребру $\Gamma_{3}^{(1)}$ соответствует укороченное уравнение

$$
\hat{f}_{3}^{(1)}(X) \stackrel{\text { def }}{=} x^{2} y^{2}-x^{4}=0
$$

с нормальным конусом $\mathbf{U}_{3}^{(1)}=\{P=\lambda(1,1), \lambda>0\}$, т.е. $\omega=1$ и $x \rightarrow \infty$. Очевидно, $\pi\left(\hat{f}_{3}^{(1)}\right)=0<2=\pi(f)$.

Согласно п. 4.6 укороченному уравнению (8.2) соответствуют два разложения (4.18)

$$
y=c_{1} x+x^{-1} \sum_{k=0}^{\infty} c_{-2 k} x^{-2 k} \stackrel{\text { def }}{=} \varphi_{l}(x), \quad l=1,2,
$$

где $c_{1}=(-1)^{l}$ и все $c_{-2 k}$ однозначно определены для фиксированного $l$. Согласно теореме 7.2 для каждого разложения (8.3) имеются две однопараметрических добавки вида (7.21). Найдем их начальные отрезки. Вычислим первую вариацию

$$
\frac{\delta f}{\delta y}=2 x^{2} y^{\prime} \frac{d}{d x}-2 x^{2} y^{\prime \prime}-2 x^{2} y \frac{d^{2}}{d x^{2}}+2 a y+2 x^{2} y .
$$

Согласно лемме 7.1 на разложении (8.3) вариация (8.4) дает оператор $\mathscr{M}(x)$. Вьгислим все те дифференциальные мономы $a_{i}(x, z)$ суммы $\mathscr{M}(x) z$, у которых векторные показатели степени $Q\left(a_{i}\right)=\left(q_{1}, 1\right)$ имеют $q_{1}>0$, и их сумму обозначим через $\mathscr{N}(x) z$. Согласно (8.4) и (8.3) получаем

$$
\mathscr{N}(x)=2 x^{2} c_{1} \frac{d}{d x}-2 c_{1} x^{3} \frac{d^{2}}{d x^{2}}+2 a c_{1} x+2 c_{1} x^{3}+2 c_{0} x .
$$

Произведем дальнейшие вычисления, используя оператор $\mathcal{N}(x)$ вместо оператора $\mathscr{M}(x)$. Уравнение $\mathscr{N}(x) z=0$ после логарифмического преобразования (7.10) и сокращения на $2 c_{1} x z$ принимает вид

$$
\tilde{h} \stackrel{\text { def }}{=} x \zeta-x^{2}\left(\zeta^{\prime}+\zeta^{2}\right)+a+x^{2}+c_{0} / c_{1}=0 .
$$


Его носитель $\mathbf{S}(\tilde{h})$ и многоугольник $\Gamma(\tilde{h})$ показаны на рис. 15 . Из него видно, что с конусом задачи $\widetilde{\mathscr{K}}=\left\{\tilde{P}=\left(\tilde{p}_{1}, \tilde{p}_{2}\right): \tilde{p}_{1}+\tilde{p}_{2}>0\right\}$ пересекаются только нормальные конусы вертикального ребра и его вершин. Но согласно следствию 7.1 укорочения, соответствуюшие вершинам, не дают подходящих решений. Вертикальному ребру соответствует укороченное уравнение $\hat{h} \stackrel{\text { def }}{=}-x^{2} \zeta^{2}+x^{2}=0$. Его решения суть

$$
\zeta=\alpha_{i}=(-1)^{i}, \quad i=1,2 .
$$

Поскольку сдвинутый носитель уравнения

$$
h(x, \zeta) \stackrel{\text { def }}{=} z^{-1} \mathscr{M}(x) z=0
$$

расположен в решетке с базисом $(2,0),(1,1)$, а сдвинутый носитель укороченного решения $(8.7)$ - это вектор $(0,1)$, то все они расположены в целочисленной решетке $\mathbb{Z}^{2}$. Поэтому соответствуюшие (8.7) разложения решений уравнения $h=0$ имеют вид

$$
\zeta=\alpha_{i}+\sum_{k=1}^{\infty} \delta_{-k} x^{-k}
$$

Вычислим коэффициент $\delta_{-1}$. Подставим $\zeta=\alpha_{i}+\delta_{-1} x^{-1}$ в уравнение (8.6) и, приравнивая нулю коэффициент при $x$, получим для $\delta_{-1}$ уравнение $\alpha_{i}-2 \alpha_{i} \delta_{-1}=0$. Из него находим, что $\delta_{-1}=1 / 2$. Согласно замечанию 7.1 в разложении $(8.8)$ все $\delta_{-k}$ суть определенные постоянные, и решения, близкие к разложениям (8.3), имеют вид

$$
y=\varphi(x)+c x^{1 / 2} \exp \left[\alpha_{i} x+\sum_{k=1}^{\infty} \gamma_{-k} x^{-k}\right]+\cdots
$$

где $c$ - произвольная постоянная, все $\gamma_{-k}$ - определенные постоянные, a $\alpha_{i}=\alpha_{1}=-1$, если $\operatorname{Re} x \rightarrow+\infty$, и $\alpha_{i}=\alpha_{2}=1$, если $\operatorname{Re} x \rightarrow-\infty$, поскольку добавка должна стремиться к нулю при $x \rightarrow \infty$. В [14] аналогично получена экспоненщиальная добавка в первом уравнении Пенлеве.

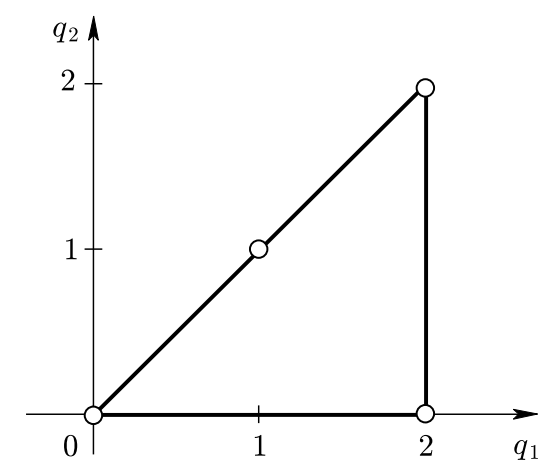

Рис. 15. 
Согласно замечанию 7.4 найдем экспоненциальную добавку к разложению (8.8) решения уравнения $\left(8.7^{\prime}\right)$. Согласно (8.4) и лемме 7.1 оператор $\mathscr{M}(x)$ есть

$$
\mathscr{M}(x)=2 x^{2} \varphi^{\prime} \frac{d}{d x}-2 x^{2} \varphi^{\prime \prime}-2 x^{2} \varphi \frac{d^{2}}{d x^{2}}+2 a \varphi+2 x^{2} \varphi
$$

где $\varphi=\varphi_{l}$, а уравнение $\left(8.7^{\prime}\right)$ есть

$$
h(x, \zeta) \stackrel{\text { def }}{=} 2 x^{2} \varphi^{\prime} \zeta-2 x^{2} \varphi^{\prime \prime}-2 x^{2} \varphi\left(\zeta^{\prime}+\zeta^{2}\right)+2 a \varphi+2 x^{2} \varphi=0 .
$$

Пусть согласно [15]

$$
\zeta_{i}(x)=\alpha_{i}+\frac{1}{2} x^{-1}+\sum_{k=1}^{\infty} \delta_{-k} x^{-k}
$$

- разложение решения этого уравнения. Положим $\zeta=\zeta_{i}(x)+w$. Тогда укорочение уравнения для $w$ имеет вид

$$
\widetilde{\mathscr{M}}(x) w=0,
$$

где согласно лемме 7.1 оператор $\widetilde{\mathscr{M}}=\delta h / \delta \zeta$ на $\zeta=\zeta_{i}(x)$, т.е.

$$
\widetilde{\mathscr{M}}(x)=2 x^{2} \varphi^{\prime}-2 x^{2} \varphi\left(\frac{d}{d x}+2 \zeta_{i}\right)
$$

После логарифмического преобразования $\xi=d \ln w / d x=d w /(w d x)$ из уравнения (8.9) получаем уравнение

$$
\tilde{h}(x, \xi) \stackrel{\text { def }}{=} w^{-1} \widetilde{\mathscr{M}} w \stackrel{\text { def }}{=} 2 x^{2} \varphi^{\prime}-2 x^{2} \varphi\left(\xi+2 \zeta_{i}\right)=0 .
$$

Его решение есть

$$
\xi_{i}=-2 \zeta_{i}+\varphi^{\prime} / \varphi=-2 \alpha_{i}+\sum_{k=2}^{\infty} \tilde{\delta}_{k} x^{-k} .
$$

Следовательно, разложения решений уравнения (8.1) имеют вид

$$
y=\varphi(x)+\exp \left[\int \zeta_{l}(x) d x+\exp \left(\int \xi_{i}(x) d x+\cdots\right)\right] .
$$

Поскольку $\zeta_{i}=\alpha_{i}+\cdots, \alpha_{i}=(-1)^{i}$ и $\xi_{i}=-2 \alpha_{i}+\cdots$, то при $|x| \rightarrow \infty$ если $\int \zeta_{i} d x \rightarrow$ $-\infty$, то $\int \xi_{i} d x \rightarrow+\infty$. Поэтому вторая добавка $\exp \int \xi_{i} d x$ отсутствует в согласии с замечанием 7.4. 


\section{Заключение}

Методами $\S \S 1$ и 3 в [22]-[26], [36] были получены новые разложения решений третьего, пятого и шестого уравнений Пенлеве, отсутствующие в обзорной книге [38].

Методами $\S \S 1,3,5$ в [33]-[35], [41] были исследованы решения обькновенных дифференциальных уравнений, к которым сводятся гидродинамические задачи о пограничных слоях на игле в потоках жидкостей разных типов.

Все изложенное в $\S \S 1-8$ переносится на систему обыкновенных дифференциальных уравнений. А именно, нахождение степенных асимптотик решений см. в [2; гл. VI, §1], [3; раздел 8], [7], [8; п. 3], вычисление их критических чисел см. в [7], [8; пा. 1, 2], [27; §2], вычисление степенных разложений решений см. в [7], [8], [27; § 2], вычисление степенно-логарифмических разложений решений см. в [16], вычисление нестепенных асимптотик решений см. в $[9 ; \S 1]$, вычисление экспоненциально малых добавок к решению см. в [17]. В [7] приведены примеры применения этой техники к системе Лоренца и к задаче Хилла.

Но главным образом эта техника применялась к системе Н. Ковалевского [39], описьвающей движения тяжелого твердого тела с закрепленной точкой в случае

$$
B \neq C, \quad x_{0} \neq 0, \quad y_{0}=z_{0}=0 .
$$

Система уравнений Н. Ковалевского имеет одну независимую и две зависимые переменные, всего три. Поэтому она исследуется с помошью трехмерной степенной геометрии, т.е. все геометрические конструкции изображаются с помощью наглядных рисунков. А именно, многогранники уравнений системы построены в [27], [28]. Степенные асимптотики решений, их критические числа и соответствующие степенные разложения решений вычислены в [28]-[32], [10]. Степенно-логарифмические разложения решений обсуждаются в $[11 ; \S 6],[12 ;$ п. 6$],[16 ; \S 3]$. На их основе делается вьвод об отсутствии дополнительного аналитического первого интеграла при $A \neq B$, при $A=B<C$ и при $A=B=22 C$. Нестепенные асимптотики решений вычислены в $[9 ; \S \S 2-5],[11 ; \S 4],[12 ;$ п. 4]. На их основе делается вывод об отсутствии дополнительного аналитического первого интеграла при $A=B=4 C / 3$ и при $A=B=4 C$. В [17] показано, что разложения решений этой системы не имеют экспоненщиальных добавок. Кроме того, в теореме 2.1 из [10] указано счетное множество значений $y=$ $A / C=B / C$, при которых все разложения решений имеют рациональные показатели степеней, и (кроме $y=4$ ) отсутствуют нестепенные асимптотики. В этих случаях можно ожидать существование дополнительного первого интеграла, содержащего рациональные степени переменных.

Обзор основных идей и результатов степенной геометрии см. в [15].

Первоначальньй вариант этой работы - препринты [13], [14]. Аннотация основных результатов дана в [18]-[21].

\section{СПИСОК ЛИТЕРАТУРЫ}

[1] А. Д. Брюно. Локальный метод нелинейного анализа дифференциальных уравнений. М.: Наука, 1979.

[2] А. Д. Брюно. Степенная геометрия в алгебраических и дифференциальных уравнениях. М.: Физматлит, 1998. 
[3] A.D. Bruno. Power geometry as a new calculus // Analysis and Applications / ed. H. G. W. Begehr, R. P. Gilbert, M. W. Wong. Dordrecht: Kluwer, 2003. P. 51-71. (Int. Soc. Anal. Appl. Comput. V. 10.)

[4] А. Д. Брюно. Степенные разложения решений одного алгебраического или дифференциального уравнения // Препринт № 63. М.: ИПМ им. М.В. Келдыша, 2000.

[5] А. Д. Брюно. Степенные разложения решений одного алгебраического или дифференциального уравнения // Докл. РАН. 2001. Т. 380. № 2. С. 155-159.

[6] А. Д. Брюно. Автомодельные решения и степенная геометрия // УМН. 2000. Т. 55. № 1. C. $3-44$.

[7] А. Д. Брюно. Степенные разложения решений системы алгебраических и дифференциальных уравнений // Препринт № 68. М.: ИПМ им. М.В. Келдыша, 2000.

[8] А. Д. Брюно. Степенные разложения решений системы алгебраических и дифференциальных уравнений // Докл. РАН. 2001. Т. 380. № 3. С. 298-304.

[9] А. Д. Брюно. Асимптотики решений обыкновенных дифференциалшных уравнений // Препринт № 40. М.: ИПМ им. М. В. Келдыша, 2002.

[10] А. Д. Брюно. Степенные свойства движений твердого тела // Докл. РАН. 2002. Т. 387. № 6. C. $727-732$.

[11] А. Д. Брюно. Анализ уравнений Эйлера-Пуассона методами степенной геометрии // Препринт № 41. М.: ИПМ им. М. В. Келдыша, 2002.

[12] А. Д. Брюно. Анализ уравнений Эйлера-Пуассона методами степенной геометрии // Механика твердого тела (Донецк). № 32, 2003. С. 3-15.

[13] А. Д. Брюно. Асимптотики и разложения решений обькновенного дифференциального уравнения // Препринт № 9. М.: ИПМ им. М. В. Келдыша, 2003.

[14] А. Д. Брюно. Асимптотически близкие решения обькновенного дифференциального уравнения // Препринт № 31. М.: ИПМ им. М. В. Келдыша, 2003.

[15] А. Д. Брюно. Асимптотическое решение нелинейных уравнений с помощью степенной геометрии // Препринт № 28. М.: ИПМ им. М.В.Келдыша, 2003.

[16] А. Д. Брюно. Разложения решений системы ОДУ // Препринт № 59. М.: ИПМ им. М. В. Келдыша, 2003.

[17] А. Д. Брюно. Асимптотически близкие решения системы ОДУ // Препринт № 58. М.: ИПМ им. М. В. Келдыша, 2003.

[18] А. Д. Брюно. Степенные асимптотики решений обыкновенного дифференциального уравнения // Докл. РАН. 2003. Т. 392. № 3. С. 295-300.

[19] А. Д. Брюно. Степенно-логарифмические разложения решений обькновенного дифференциального уравнения // Докл. РАН. 2003. Т. 392. № 4. С. 439-444.

[20] А. Д. Брюно. Нестепенные асимптотики решений обыкновенного дифференциального уравнения // Докл. РАН. 2003. Т. 392. № 5. С. 586-591.

[21] А. Д. Брюно. Асимптотически близкие решения обькновенного диффференциального уравнения // Докл. РАН. 2003. Т. 393. № 4. С. 448-452.

[22] А. Д. Брюно, И. В. Чухарева. Степенныые разложения решений шестого уравнения Пенлеве // Препринт № 49. М.: ИПМ им. М. В. Келдьша, 2003.

[23] А. Д. Брюно, И. В. Горючкина. Разложения решений шестого уравнения Пенлеве // Докл. РАН. 2004. Т. 395. №6. С. 733-737.

[24] А. Д. Брюно, А. В. Гриднев. Степенньше и экспоненциальные разложения решений третьего уравнения Пенлеве // Препринт № 51. М.: ИПМ им. М. В. Келдьша, 2003.

[25] А. Д. Брюно, Е.С. Карулина. Степенньше разложения решений пятого уравнения Пенлеве // Препринт № 50. М.: ИПМ им. М. В. Келдьша, 2003.

[26] А. Д. Брюно, Е. С. Карулина. Разложения решений пятого уравнения Пенлеве // Докл. РАН. 2004. Т. 395. № 4. С. 439-444.

[27] А. Д. Брюно, В.В.Лунев. Модифицированная система уравнений движения твердого тела // Препринт № 49. М.: ИПМ им. М.В.Келдыша, 2001.

[28] А. Д. Брюно, В.В.Лунев. О вычислении степенных разложений модифицированных движений твердого тела // Докл. РАН. 2002. Т. 386. №1. С. 11-17. 
[29] А. Д. Брюно, В.В.Лунев. Локальные разложения модифицированных движений твердого тела // Препринт № 73. М.: ИПМ им. М.В.Келдыша, 2001.

[30] А. Д. Брюно, В.В. Лунев. Асимптотические разложения модифицированных движений твердого тела // Препринт № 90. М.: ИПМ им. М.В. Келдыша, 2001.

[31] А. Д. Брюно, В.В.Лунев. Свойства разложений модифицированных движений твердого тела // Препринт № 23. М.: ИПМ им. М.В.Келдыша, 2002.

[32] А. Д. Брюно, В.В.Лунев. Семейства степенных разложений модифиццированных движений твердого тела // Докл. РАН. 2002. Т. 387. № 3. С. 297-303.

[33] А. Д. Брюно, Т. В. Шадрина. Об осесимметричном обтекании иглы вязкой несжимаемой жидкостью // Докл. РАН. 2002. Т. 387. № 5. С. 589-595.

[34] А. Д. Брюно, Т. В. Шадрина. Осесимметричный пограничный слой на игле // Препринт № 64. М.: ИПМ им. М. В. Келдыша, 2003.

[35] А. Д. Брюно, Т. В. Шадрина. Осесимметричньй пограничный слой на игле // Докл. РАН. 2004. Т. 394. № 3. С. 298-304.

[36] А. Д. Брюно, Ю. В. Завгородняя. Степенныые ряды и нестепенные асимптотики решений второго уравнения Пенлеве // Препринт № 48. М.: ИПМ им. М.В.Келдьша, 2003.

[37] Y.F. Chang, J. M. Greene, M. Tabor, J. Weiss. The analytic structure of dynamical systems and self-similar natural boundaries // Phys. D. 1983. V. 8. № 1-2. P. 183-207.

[38] V. I. Gromak, I. Laine, S. Shimomura. Painlevé Differential Equations in the Complex Plane. Berlin: de Gruyter, 2002. (de Gruyter Stud. Math. V. 28.)

[39] N. Kowalewski. Eine neue partikulare Losung der Differenzialgleichungen der Bewegung eines schweren starren Korpers um einen festen Punkt // Math. Ann. 1908. V. 65. P. 528-537.

[40] Н. Х. Розов. Пенлеве уравнение // Математическая энциклопедия. Т. 4. М.: Советская Энциклопедия, 1984. С. 233-234.

[41] Т. В. Шадрина. Об осесимметричном обтекании иглы вязкой несжимаемой жидкостью // Препринт № 36. М.: ИПМ им. М.В. Келдыша, 2002.

[42] В.М. Тихомиров. Фреше производная // Математическая энциклопедия. Т. 5. М.: Советская Энциклопедия, 1985. С. 666.

Институт прикладной математики им. М.В.Келдьша РАН

Поступила в редакцию

E-mail: bruno@keldysh.ru

07.11 .2003 